\title{
A two-dimensional variational analysis method for NSCAT ambiguity removal: Methodology, sensitivity, and tuning*
}

\author{
R. N. Hoffman ${ }^{\dagger}$, S. M. Leidner ${ }^{\dagger}$, J. M. Henderson ${ }^{\dagger}$, \\ R. Atlas ${ }^{\ddagger}$, J. V. Ardizzone ${ }^{\S}$, and S. C. Bloom ${ }^{\S}$
}

September 20, 2001

* Corresponding author address: Dr. Ross N. Hoffman, Atmospheric and Environmental Research, Inc., 131 Hartwell Avenue Lexington, MA 02421-3126 Email: rhoffman@aer.com.

${ }^{\dagger}$ Atmospheric and Environmental Research Inc., Lexington, MA 02421

$\ddagger$ Data Assimilation Office, NASA Goddard Space Flight Center, Greenbelt, Maryland

${ }^{\S}$ General Sciences Corp, a Subsidiary of Science Applications International Corporation

Submitted to Journal of Atmospheric and Oceanic Technology. 


\begin{abstract}
In this study, we apply a two-dimensional variational analysis method ( $2 \mathrm{~d}$-VAR) to select a wind solution from NASA Scatterometer (NSCAT) ambiguous winds. 2d-VAR determines a "best" gridded surface wind analysis by minimizing a cost function. The cost function measures the misfit to the observations, the background, and the filtering and dynamical constraints. The ambiguity closest in direction to the minimizing analysis is selected.

2d-VAR method, sensitivity and numerical behavior are described. 2d-VAR is compared to statistical interpolation (OI) by examining the response of both systems to a single ship observation and to a swath of unique scatterometer winds. 2d-VAR is used with both NSCAT ambiguities and NSCAT backscatter values. Results are roughly comparable. When the background field is poor, 2d-VAR ambiguity removal often selects low probability ambiguities. To avoid this behavior, an initial 2d-VAR analysis, using only the two most likely ambiguities, provides the first guess for an analysis using all the ambiguities or the backscatter data.

$2 \mathrm{~d}-\mathrm{VAR}$ and median filter selected ambiguities usually agree. Both methods require horizontal consistency, so disagreements occur in clumps, or as linear features. In these cases, 2d-VAR ambiguities are often more meteorologically reasonable and more consistent with satellite imagery.
\end{abstract}




\section{Introduction}

Scatterometers are active radars designed to measure the backscatter or normalized radar cross section (NRCS) from the earth's surface at moderate incidence angles. The advantage of using moderate incidence angles $\left(20-70^{\circ}\right)$ is that the major mechanism for scattering is then Bragg scattering from centimeter-scale waves, which are, in most conditions, in equilibrium with the local wind. Although the scatterometer winds are usually provided as neutral winds at some reference height, the measurement is physically most closely connected with surface stress (Brown 1986 [7]).

Scatterometers have been mounted on stationary platforms, aircraft and satellites. Since 1991, satellite-borne scatterometers have provided a wealth of wind data over the world ocean. Previous satellite scatterometers include Seasat-A Satellite Scatterometer (SASS) in 1979; the active microwave instruments (AMI) on the first and second European Remote Sensing satellites (ERS-1 and ERS-2), during 1991-2000; and the NASA Scatterometer (NSCAT) on the first Advanced Earth Observing Satellite (ADEOS-1) during 1996-1997. Currently and since 1999, SeaWinds on QuikSCAT has been producing useful data.

Scatterometers measure wind speed very accurately; Freilich and Dunbar (1999 [15]) report that NSCAT measured wind speed to within $1.3 \mathrm{~ms}^{-1}$ in an $r m s$ sense, while Stoffelen and Anderson (1997 [55]) suggest that the ERS-1 measurement error standard deviation is actually only $0.5 \mathrm{~ms}^{-1}$. However, the wind direction from scatterometers is not uniquely determined. Wind speed and direction are inferred from a number of closely colocated (both temporally and spatially) radar measurements from a number of different azimuth angles. The measured NRCS, denoted $\sigma^{0}$, varies with the relative azimuth angle between the antenna and wind direction. An "upwind-crosswind" variation exists because small, wind-generated gravity waves on the ocean surface reflect more of the radar signal when wave crests are perpendicular (i.e., "upwind") to the radar antenna than when wave crests are parallel (i.e., "crosswind"). The "upwind-downwind" signal is very small, because when fetch is not limited, the orientation and amplitude of these waves is nearly the same if the wind direction is shifted by $180^{\circ}$. 
Typically several $\sigma^{0}$ measurements are grouped into 25 or $50 \mathrm{~km}$ wind vector cells (WVCs) for wind retrieval. Modeled $\sigma^{0}$ values are computed from a geophysical model function which requires spacecraft position and orientation (known) and wind speed and direction (unknowns) as inputs. The normalized squared differences between measured and modeled $\sigma^{0}$ values are summed as part of the objective function of a maximum likelihood estimator (MLE). Then, wind speed and direction are varied to minimize the difference between measured and modeled $\sigma^{0}$ values. However, over the range of possible wind speeds and directions (i.e., $0-50 \mathrm{~ms}^{-1}$ and $0-360^{\circ}$ ), the MLE produces from two to six local maxima, and it is not possible to determine the wind uniquely from the MLE alone. The wind vectors corresponding to these maxima are called "ambiguities". For operational numerical weather prediction (NWP), and for other purposes it is necessary to choose one ambiguity at each location in a meteorologically consistent manner. Once we have chosen a single ambiguity, we call the result a "unique" wind.

Median filter techniques have been used for ambiguity removal, but may fail at swath edges and in regions of strongly curved or sheared flow. For NSCAT, the Jet Propulsion Laboratory (JPL) used a median filter initialized by the ambiguity with the highest MLE. This method was completely objective and autonomous. Later, better performance was obtained by initializing the scatterometer wind field with the ambiguity closest to the operational gridded surface wind field analysis from the National Centers for Environmental Prediction (NCEP, formerly the National Meteorological Center or NMC). This product is referred to as the nudged wind product since an outside source is used to initialize (or "nudge") the median filter. This method is also completely objective but depends on information in addition to the scatterometer measurements. The performance of the median filter method has been quite successful (Gonzales and Long 1999 [18]) but is not based on any physical model of wind flow. Consequently, the median filter occasionally selects physically unrealistic winds. Patches of unrealistic winds in the scatterometer data might have a disastrous impact on the quality of analyses and NWP forecasts. Careful quality control (QC) is required, of course, but is problematic if the model winds were used in the ambiguity removal. 
This paper reports on the use of a two-dimensional variational analysis method (2d-VAR) for ambiguity removal. 2d-VAR generates a gridded surface wind analysis by minimizing an objective function, which is a weighted sum of several constraints on the difference between the analysis and the background, and between the analysis and the data. While the "nudged" median filter uses a priori information for initialization, 2d-VAR uses such information both for initialization and as part of the background constraints. 2d-VAR was originally described by Hoffman (1982 [22]) to analyze Seasat scatterometer (SASS) data. In this first formulation, only observations from conventional platforms (ships, buoys, radiosondes) and from SASS were combined with an a priori or background wind field. 2d-VAR was extended to include smoothness (i.e. filtering) constraints and a dynamical constraint by Hoffman (1984, hereafter H84 [23]).

Since H84 2d-VAR has undergone several developments. These include:

- Optional use of 12 point bicubic interpolation to the "obs" locations ( $\S \mathrm{B})$.

- Use of $\sigma^{0}$ values $(\S 6 \cdot a \cdot 1)$.

- Reformulation of the constraint terms $(\S 3 . b)$.

$2 \mathrm{~d}-\mathrm{VAR}$ may combine both wind retrieval and ambiguity removal by analyzing the $\sigma^{0}$ data directly. For both ERS-1,2 and NSCAT, in addition to the possibility of analyzing $\sigma^{0}$ data, the ambiguities may be treated in the same manner as SASS data were treated by H84, or unique winds may be treated as buoy "obs". 2d-VAR has been used to produce several long running data sets for oceanographic research. First, 2d-VAR was adapted for use with time binned wind observations by FSU researchers including Legler et al. (1989 [33]), Meyers et al. (1994 [38]), Jones et al. (1995 [28]), and Pegion et al. (2000 [45]). Later, 2d-VAR was extended to SSM/I wind magnitude data by Atlas et al. $(1991,1996$ [2, 3]). We will review the $2 \mathrm{~d}-\mathrm{VAR}$ formulation in section $\S 3$, and extensions for scatterometer data in $\S 6$.

2d-VAR, in common with other 3d-VAR and 4d-VAR systems, is based on a Bayesian viewpoint. In fact the $2 \mathrm{~d}$-VAR cost function may be written as $J=J_{b}+J_{o}$, where

- $J$ is the total cost function. 
- $J_{b}$ is the background cost function.

- $J_{o}$ is the "obs" cost function.

Since $J$ is a function of the surface wind field, minimizing $J$ produces a gridded surface wind analysis that simultaneously closely fits the data and background (H84). As in any analysis method, specification of the errors of the data and the background are vitally important. Our implementation of 2d-VAR employs a heuristic model of forecast error statistics. $J_{b}$ is defined below in (3) as the weighted sum of several constraints. Each constraint function, $J_{m}$, is a scalar which measures the the difference between the analysis and background using the $m$ th constraint. The constraints include filtering and dynamical consistency. The combination of these constraints mimicks the usual $J_{b}$ term in 3d-VAR or 4d-VAR (e.g. Thépaut et al. 1993 [57]). Therefore 2d-VAR as described here may be considered to be a specialization of 3d-VAR to ocean surface wind. Wahba and Wendelberger (1980 [58]) showed that under certain conditions $3 \mathrm{~d}-\mathrm{VAR}$ and $\mathrm{OI}$ are the same. Therefore $2 \mathrm{~d}-\mathrm{VAR}$ is closely related to $\mathrm{OI}$ as well. Although the formulation of $2 \mathrm{~d}$-VAR described here is very different from standard OI or 3d-VAR, analysis results can be made similar by appropriate choice of lambda weights $\left(\lambda_{i}\right.$, multiplying factors which control how much each function contributes to the total objective function). (See $\S 5$.) Thus $2 \mathrm{~d}$-VAR is a form of OI in which the correlations are parameterized with a small number of degrees of freedom. After subjective tuning, the 2d-VAR heuristic model of forecast error statistics produces results similar to conventional error models, but has the potential advantage of being specified by a small number of parameters. In principle, these parameters might vary with geographic location, with synoptic situation and might even be tuned online, using current or recent data. As a result, 2d-VAR is an ideal candidate for on-line estimation of parameters as Dee et al. $(1998$ [11, 12]) have proposed. This feature of 2d-VAR has been exploited by Pegion et al. (2000 [45]) to objectively tune these parameters. In the work described here, these parameters are chosen subjectively based on experimentation. However some of our results address how to tune these parameters to get similar results when changing the analysis resolution, data density, or data representation (see $\S 7$.) 
The outline of the rest of this paper is the following. First we define the NSCAT data and the nominal processing of the NSCAT data $(\S 2)$. We then describe $2 \mathrm{~d}-\mathrm{VAR}$ for conventional data (§3). Experiments with a single ship observation then illustrate the numerical behavior and the sensitivity of $2 \mathrm{~d}-\mathrm{VAR}(\S 4)$. These experiments demonstrate that the subjectively tuned 2d-VAR approximates OI. Idealized OI experiments with a swath of unique NSCAT winds are presented to demonstrate the close relationship between $2 \mathrm{~d}-\mathrm{VAR}$ and $\mathrm{OI}(\S 5)$. The use of $2 \mathrm{~d}$-VAR for ambiguity removal for NSCAT is then described $(\S 6)$. Experiments using ambiguities and $\sigma^{0}$ values are compared. With proper tuning the results are similar $(\S 7)$. We have improved the quality of our ambiguity removal by using a first stage in which only "dual-ambiguities" are processed $(\S 8)$. Examples of our dual-QC method in 2d-VAR are shown in $\S 8$. a. Then $\S 9$ is a conclusion. In a companion article (Henderson et al.2001 [21]) the dual-QC 2d-VAR is applied to the entire NSCAT mission and is tested as an alternative to the standard ambiguity removal schemes in impact experiments using the GEOS-2 data assimilation system.

\section{NSCAT data}

The focus of this study are winds retrieved from NSCAT. NSCAT was launched aboard the ADEOS-1 spacecraft on 16 August 1996 from Tanegashima Space Center in Japan. Unfortunately, after only nine months of operation, the ADEOS-1 solar power array failed. ADEOS-1 had a nearly polar Sun-synchronous orbit at a height of $\sim 800 \mathrm{~km}$, with a period of $\sim 100$ minutes.

NSCAT made observations covering two $600 \mathrm{~km}$ swaths, one on either side of the spacecraft, separated by a gap of $\sim 330 \mathrm{~km}$ corresponding to low incidence angles. On each side of the spacecraft, NSCAT operated three $3 \mathrm{~m}$ long, stick-like antennas at $13.995 \mathrm{GHz}$. The foreward and aft antennas were vertically polarized, while the mid antenna was both vertically and horizontally polarized. Thus NSCAT made observations in sequence from eight beams, i.e. from eight combinations of antennas and polarizations. While the foreward 
and aft antennas are separated by $90^{\circ}$ as in the ERS-1 design, the angles between the mid antenna and the others are 20 and $70^{\circ}$, whereas in the ERS-1 design these angles are $45^{\circ}$. Complete descriptions of the NSCAT antenna subsystems, resolution, registration of observations, measurement sequencing, hardware, and ground system are provided by Naderi et al. (1991 [39]).

In the ground processing, the individual backscatter observations associated with the different beams are organized by a grid oriented along the satellite track. Nominally there are 4 or 16 backscatter observations in a 25 or $50 \mathrm{~km}$ WVC. The WVC coordinates along, and perpendicular to, the satellite track are called row and cell, respectively. Each cell is associated with a nearly constant incidence angle for each antenna, with the values being equal for the fore and aft antennas. Wind ambiguities are retrieved for each WVC containing a sufficient number of quality controlled backscatter observations. The retrieved winds maximize a likelihood function locally. The likelihood function in turn depends on the geophysical model function to compare a candidate wind to the backscatter observations (Chi and Li 1988, Offiler $1994[8,44])$. Examples of the likelihood function are described in $\S 6 . a .1$.

Geophysical model functions are empirical relationships used to relate the backscatter to the geophysical parameters, and are derived from colocated observations (Jones et al. 1977 [30]). In current model functions, the backscatter depends nonlinearly on wind speed and direction. Models developed from aircraft flights have been used as baseline model functions for satellite instruments. However substantial post-launch modifications and refinements were needed for SASS (Boggs 1981 [5]) and for ERS-1 (Offiler 1994, Stoffelen and Anderson 1997 [44, 55]). The prelaunch NSCAT model function, denoted NSCAT-0 or SASS2, was developed by Wentz et al. (1984 [60]) from the Seasat data set by matching the statistics of the observed $\sigma^{0}$ and those simulated assuming that the ocean surface winds follow a one parameter Weibull distribution (Conradsen et al. 1984 [9]). The fitting procedure used six parameters at each polarization and incidence angle. The first postlaunch NSCAT model function, denoted NSCAT-1, was developed using a combination of SSM/I, NCEP and European Centre for Medium-Range Weather Forecasts (ECMWF) winds colocated with 
NSCAT $\sigma^{0}$ observations. A further refinement, denoted NSCAT-2, has been developed by Wentz and Smith (1999 [61]), based on colocations with SSM/I and ECMWF winds.

There have been many theoretical attempts to derive model functions for scatterometers but they have not been as successful as the purely empirical model functions. A moments consideration of the complexity of the ocean surface under moderate to high wind conditions suggests that a purely theoretical approach is a difficult and ambitious undertaking. Recent examples include Janssen (1994 [27]) and Romeiser et al. (1997 [49, 50]).

Note that the model function relates wind speed and direction to the backscatter. In this calculation the geometry of the observation is taken as a given. To retrieve scatterometer wind information, several backscatter measurements at the same earth location, but with different viewing geometries, must be used. The winds are obtained by optimally fitting these data.

Because of the nonlinearity of the model function and the various sources of error, several wind vectors consistent with the backscatter observations are usually found (Price, 1976 [47]). (See §6.a.) These multiple wind vectors are called aliases in the early literature and are now generally referred to as ambiguities. With two beams, SASS typically retrieved four ambiguities. With additional beams, NSCAT and ERS often retrieve just two ambiguities with directions approximately $180^{\circ}$ out of phase. The ambiguities are ordered by likelihood. The first ambiguity is the ambiguity with the highest likelihood; it is most consistent with the backscatter data. The second ambiguity is usually of nearly equal speed and consistency, but of nearly opposite direction.

Since the wind retrieval is performed for each WVC individually and since there is little to distinguish the first two ambiguities, a wind field of first ambiguities will generally be very irregular with many wind reversals. Spatial filtering can be used to produce a horizontally consistent wind field and resolve the directional ambiguity. A variety of approaches have been used. These include the work of Hoffman (1984 [23]), Schroeder et al. (1985 [51]), Schultz (1990 [52]), Shaffer et al. (1991 [53]), Wentz (1991 [59]), Badran et al. (1991 [4]), Offiler (1992 [43]), Thépaut et al. (1993 [56]), Long (1993 [35]), Stoffelen and Anderson 
(1997 [54]), Jones et al. (1999 [29]), Figa and Stoffelen (2000 [13]), and de Vries and Stoffelen $(2000[10])$. Simulation studies for NSCAT established that a median filter operating autonomously, i.e. with no a priori information, would provide excellent ambiguity selection (Schultz 1991, Shaffer et al. $1991[52,53]$ ). The median filter must be initialized or seeded with some initial choice of ambiguities. In autonomous mode the seed is the first ambiguity. In practice this choice did not perform as well as expected. For the nudged median filter, the seed is the ambiguity closest to a background obtained from an operational analysis or short term forecast. No further reference to the background is made. At the end of the process the chosen winds are consistent with backscatter observations in the WVC and horizontally consistent with neighboring WVCs, but are not necessarily close to the background. Differences between autonomous and nudged results show that the median filter has multiple solutions. Thus spatial filtering does not resolve all ambiguity in the sense that multiple horizontally consistent wind fields are plausible.

It should be noted that there are several NSCAT data products available. These differ in resolution (25 or $50 \mathrm{~km}$ ), geophysical model function (NSCAT-0, NSCAT-1, or NSCAT-2), and ambiguity selection procedure (autonomous or nudged median filter). All JPL NSCAT data sets use the median filter described by Shaffer et al. (1991 [53]). The $25 \mathrm{~km}$ resolution data sets based on the NSCAT-2 model function and the nudged median filter are the most current. Some of the preliminary results presented here however use other versions of the NSCAT data.

\section{2d-VAR method}

2d-VAR finds the minimum of an objective function. The objective function depends on the surface wind field. Given any wind field the objective function measures the difference between the given field and the observations, and between the given field and the a priorior background wind field. The minimizer of the objective function is therefore the wind field which optimally fits the observed and a priori data. 
The order of the steps taken in 2d-VAR is flexible and the subject of ongoing tuning. The nominal and minimal setup is to:

1. Define the geometry of the analysis grid, and on this grid define the background wind field.

2. Set the inital estimate for the analysis equal to the background.

3. Read the "obs" data sets, making necessary conversions and performing gross quality control (QC).

4. QC the "obs" based on simulated values from the background.

5. Minimize the objective function $J$.

6. Write the analysis wind field and simulated "obs" values from the analysis.

This basic operation may be modified in several ways.

- The initial guess may be an arbitrary wind field.

- After a preliminary analysis, the analysis and background can be interpolated to a finer grid. The minimization then restarts, using the newly interpolated analysis as the initial estimate of the fine scale analysis.

- After a preliminary analysis, all data may be subjected to a second QC. This accepts some of the data which were initially rejected but are corroborated by near-by data. The minimization might then restart.

In $\S 8$ a small modification of this basic setup provides the dual QC approach.

In order to minimize the objective function $J$ we must be able to calculate it for any physically reasonable value of the control variable vector $X$, which here represents the surface wind field. In the current formulation $\boldsymbol{X}$ contains those values of the gridded wind components $(u, v)$ which are independent and which are free to vary. Some points in the gridded wind field are not independent. In a global field points at longitudes $0^{\circ}$ and $360^{\circ}$ 
are the same. Not all points in the gridded wind fields are allowed to vary. If there are fixed points, they are not part of the control vector. The fixed points are nominally set equal to the background, but in general they are arbitrary. In particular for multiprocessing using domain decomposition the fixed points may be obtained from another processor's previously computed solution. A detailed description of the gridded wind field representation is given in $\S \mathrm{A}$.

Efficient minimization also requires the calculation of the gradient of $J$, i.e. $\partial J / \partial \boldsymbol{X}$. This is accomplished by the adjoint of the routines which calculate $J$ (Hoffman et al. 1992 [26]). The minimization procedure we use is a version of the limited-memory quasi-Newton algorithm (Liu and Nocedal 1989 [34]), which was described and evaluated by Navon and Legler (1987 [40]). The stopping criteria is either

$$
|G| \leq \epsilon \max (1,|\boldsymbol{X}|)
$$

or, until $N_{f}$ function evaluations, whichever occurs first. Here

- $G$ is the gradient of $J$ with respect to $X$.

- $\epsilon$ is the gradient test stopping parameter.

- $N_{f}$ is the function call stopping parameter.

We have been unsuccessful at preconditioning the minimization. Typically several hundred iterations are required to satisfy our very strict convergence criterion. In a typical experiment the rms difference in the $u$ or $v$ wind components between the solution after 250 and after 225 function calls is $\sim 0.02 \mathrm{~ms}^{-1}$, with maximum differences of $\sim 0.5 \mathrm{~ms}^{-1}$. The reason for the slow convergence is due to the step by step expansion of the region of influence of the data (see Fig. 5). A regridding process speeds convergence ( $\S 4$ b. . 2 ).

The $J$ used in this study may be written in the form,

$$
J=J_{b}+J_{o}
$$

where

$$
J_{b}=\lambda_{\mathrm{VWM}} J_{\mathrm{V} \mathrm{WM}}+\lambda_{\mathrm{LAP}} J_{\mathrm{LAP}}+\lambda_{\mathrm{DIV}} J_{\mathrm{DIV}}+\lambda_{\mathrm{VOR}} J_{\mathrm{VOR}}+\lambda_{\mathrm{DYN}} J_{\mathrm{DY} N}
$$


and

$$
J_{o}=\lambda_{\mathrm{CONV}} J_{\mathrm{CONV}}+\lambda_{\mathrm{AMB}} J_{\mathrm{AMB}}+\lambda_{\mathrm{SPD}} J_{\mathrm{SPD}}+\lambda_{\mathrm{NRCS}} J_{\mathrm{NRCS}}
$$

Here, the weights, denoted $\lambda_{i}$, control the amount of influence each constraint has on the final analysis. As mentioned in the Introduction, the $\lambda_{i}$ are chosen subjectively in this study, but techniques to tune them objectively have been developed. Values of the $\lambda_{i}$ which were used for various experiments are reported below. It should be noted that these are weak constraints and therefore the $\lambda_{i}$ are not Lagrangian multipliers. Table 1 contains a summary of the constraints used. Details of the formulation follow, or are given in the Appendices.

\section{3.a "Obs" functions}

In $2 \mathrm{~d}-\mathrm{VAR}$, the misfit between data and analysis is measured by a objective function defined as a sum of squared errors over all data locations. Multiplying factors, called lambda weights and denoted by $\lambda_{i}$, control how much each function contributes to the total objective function. For example, we might divide ships into special research ships and all others and buoys into Tropical Atmosphere-Ocean (TAO) buoys and all others, or we might lump all these data into the conventional platform class. Similarly we might divide the SSM/I data depending on the DMSP spacecraft designation (e.g., F8), or keep them all together. We expect the minimizing analysis to fit the data more closely for a given data type as the $\lambda$ weight for that data type is increased.

In general, the total "obs" function, $J_{o}$ is the weighted sum of individual "obs" operators for each data class, but here we report experiments with only one type of data and some of the

$$
\lambda_{o}=\left(\lambda_{\mathrm{CONV}}, \lambda_{\mathrm{AMB}}, \lambda_{\mathrm{SPD}}, \lambda_{\mathrm{NRCS}}\right)
$$

are set to zero. Usually the "obs" function is given by the sum of squared differences between simulated (i.e., calculated from the analysis) and data values normalized by the estimated "obs" (and representativeness) error(s). For example,

$$
J_{\mathrm{CONV}}=\sum \frac{\left(u_{a}-u_{o}\right)^{2}}{s_{u}^{2}}+\frac{\left(v_{a}-v_{o}\right)^{2}}{s_{u}^{2}} .
$$


Here

- $\left(u_{a}, v_{a}\right)$ is the analyzed wind interpolated to the "obs" locations as described in $\S \mathrm{B}$.

- $\left(u_{o}, v_{o}\right)$ is the observed wind.

- $s_{u}$ is the wind component error standard deviation.

If "obs" errors within the data class are correlated in a known way, these correlations can be accounted for as described by Thépaut et al. (1993[56]), by setting $J_{\text {Conv }}=Z^{T} \mathrm{O}^{-1} \boldsymbol{Z}$. Here

- $Z$ is the vector of normalized departures. $Z$ would contain an entry for each wind component of each "obs".

- $\mathrm{O}$ is the matrix of observation error correlations.

In the 2d-VAR experiments reported here, "obs" error correlations are ignored. Currently $s_{u}$ is taken to be a constant $1 \mathrm{~ms}^{-1}$ and knowledge of the "obs" error standard deviation is absorbed into $\lambda_{\text {Conv }}$. The special "obs" functions for scatterometer data are described later in $\oint 6 . \mathrm{a}$.

\section{3.b Background constraints}

As given in (3), the background function, $J_{b}$ is a weighted sum of several constraints, each providing a measure of the difference between the analysis and background wind field. The simplest constraint measures the square of the vector wind magnitude:

$$
J_{\mathrm{VWM}}=\frac{1}{S_{\mathrm{VWM}}} \int_{A}\left(V_{a}-V_{b}\right)^{2} d A
$$

The effect of $J_{\mathrm{Vwm}}$ is to constrain the size of the analysis increments.

The scaling parameter $S_{\mathrm{vwm}}$ makes $J_{\mathrm{vwm}}$ nondimensional and of convenient magnitude. [If these were not concerns $S_{\mathrm{vwm}}$ might be absorbed into $\lambda_{\mathrm{vwm}}$ since the adjustable parameter $\lambda_{\text {VwM }}$ multiplies $J_{\text {VwM }}$ in (3).] We define $S_{\text {VwM }}$ in terms of typical velocity and length scales,

$$
S_{\mathrm{VWM}}=V^{2} L^{2}=L^{4} / T^{2}
$$


Here

- $L$ is the length scale $\left[10^{6} \mathrm{~m}=10^{3} \mathrm{~km}\right]$.

- $V$ is the velocity scale $\left[10 \mathrm{~ms}^{-1}\right]$.

And from these we define a time scale for later use:

- $T$ is the time scale $\left[L / V=10^{5} \mathrm{~s}\right]$.

The other background constraints which are summarized in Table 1 are given by

$$
\begin{gathered}
J_{\mathrm{LAP}}=T^{2} \int_{A}\left[\nabla^{2}\left(u_{a}-u_{b}\right)\right]^{2}+\left[\nabla^{2}\left(v_{a}-v_{b}\right)\right]^{2} d A, \\
J_{\mathrm{DIV}}=\frac{T^{2}}{L^{2}} \int_{A}\left[\nabla \cdot\left(V_{a}-V_{b}\right)\right]^{2} d A, \\
J_{\mathrm{VOR}}=\frac{T^{2}}{L^{2}} \int_{A}\left[\nabla \cdot k \times\left(V_{a}-V_{b}\right)\right]^{2} d A, \text { and } \\
J_{\mathrm{DYN}}=\frac{T^{4}}{L^{2}} \int_{A}\left(\left.\frac{\partial \zeta}{\partial t}\right|_{a}-\left.\frac{\partial \zeta}{\partial t}\right|_{b}\right)^{2} d A .
\end{gathered}
$$

Here

- $\left(u_{b}, v_{b}\right)$ is the background wind.

- $\zeta$ is the relative vorticity.

- $k$ is the unit vertical vector.

Details of the calculation of $J_{b}$ are given in the appendix $(\S \mathrm{C})$.

\section{Response to a single ship observation}

Experiments with a single conventional wind observation are presented in this section. These experiments are similar to those in H84. However results of the current experiments are somewhat at odds with the earlier conclusions because H84 was overly optimistic about the convergence of the 2d-VAR minimization and did not always use sufficient iterations in high resolution experiments. Here we show that 
- The 2d-VAR solution (after sufficient iterations) is only weakly dependent on grid resolution. (Fig. 3.)

- The 2d-VAR solutions (each of which is the end of a convergent iteration) converge as resolution increases. (Fig. 4.)

- The convergence rate of the $2 \mathrm{~d}-\mathrm{VAR}$ minimization as presently implemented is slow, especially as resolution increases. (Fig. 5.)

- For practical purposes, a high resolution 2d-VAR analyses should be obtained as the last in a series of $2 \mathrm{~d}-\mathrm{VAR}$ analyses at increasing resolution, each initialized by the previous one.

- At high resolution, the effect of interpolation errors becomes noticeable and bicubic interpolation should be used. (Fig. 9.)

- The sum of two single "obs" solutions is qualitatively the same as the solution for the two "obs" together. (Fig. 10.)

\section{4.a Methodology}

Most of the results presented in this section are based on 2d-VAR analyses for a single ship at $42 \mathrm{~N}, 50 \mathrm{~W}$, observing $30 \mathrm{~ms}^{-1}$, from $210^{\circ}$. For these experiments the background field is taken to be totally calm. The resulting analyses are essentially Green's functions, i.e., the response of the analysis to a single impulse.

All analyses are done on a grid large enough that boundary effects should be small. The grid runs from $290 \mathrm{E}$ to $330 \mathrm{E}$, and from $24 \mathrm{~N}$ to $60 \mathrm{~N}$. For graphical output we thinned (or interpolated) the analysis grid to one degree resolution in the window $302 \mathrm{E}$ to $322 \mathrm{E}$ and $35 \mathrm{~N}$ to $50 \mathrm{~N}$.

Most results are for

$$
\lambda=\left(\lambda_{\mathrm{CONV}}, \lambda_{\mathrm{VWM}}, \lambda_{\mathrm{LAP}}, \lambda_{\mathrm{DNV}}, \lambda_{\mathrm{VOR}}, \lambda_{\mathrm{DYN}}\right)^{T}=(80,1,4,16,4,64)^{T}
$$


and the single ship "obs". These $\lambda$ values are equivalent to the nominal case we have used before except that $J_{\mathrm{vw}}$ is given $1 / 4$ of the weight used by $\mathrm{H} 84$.

\section{4.b Results}

Results for the current nominal case are given in Fig. 1. The response is nearly $20 \mathrm{~ms}^{-1}$ at the observation location. The response is elongated in the direction of the wind observation, and at sufficient distance in the perpendicular direction, there is a return flow, most significantly to the northwest.

The qualitative response for the nominal case is similar the response of ordinary statistical (or optimal) interpolation to a single wind observation. Fig. 2 shows the optimal interpolation result for the same situation, assuming the wind component "obs" standard deviation for ships is $2.2 \mathrm{~ms}^{-1}$ and using NOGAPS forecast error statistics (Goerss and Phoebus 1993 [17]). Compared to the nominal case, these results are more symmetric, with the influence of the data extended further in the transverse direction and less in the longitudinal direction.

\section{4.b.1 Effect of grid resolution}

Runs were made with grid increments of $2,1,1 / 2,1 / 4$ and $1 / 8^{\circ}$ using various methods of initialization and stopping criteria.

For resolutions from 1 to $1 / 8^{\circ}$ (Fig. 3 shows the results for 2 to $1 / 4^{\circ}$ ) the solutions are nearly the same. That is, if everything else is fixed (weights, data, domain, ...) $2 \mathrm{~d}-\mathrm{VAR}$ produces the same answer independent of resolution, if it is allowed to iterate sufficiently. The $2^{\circ}$ solution (Fig. 3) has somewhat jagged contours and exhibits less return flow, but is similar to the higher resolution solutions.

The implication of these findings is that for constraints like those we have been using, anything finer than a $1^{\circ}$ resolution is unnecessary and only relatively small errors are introduced by using $2^{\circ}$ resolution. Using a higher resolution $2 \mathrm{~d}-\mathrm{VAR}$ grid would not produce a higher resolution analysis, only more grid points. Weaker background constraints should produce an analysis more closely fitting high resolution data. 
In Fig. 4 differences with respect to the $1 / 8^{\circ}$ solution are displayed. Clearly, the solutions obtained are approaching a limit as the resolution is increased. Differences are concentrated at the "obs" location. These differences are small and in obtaining these results it is critical that the $2 \mathrm{~d}-\mathrm{VAR}$ minimization has converged. Doubling the resolution, quadruples the degrees of freedom, quadruples the computation effort per iteration and quadruples the number of iterations required to achieve comparable accuracy, thereby increasing the memory requirement by a factor of 4 and the timing requirement by a factor of 16 . At high resolution, convergence to the true solution is quite slow, and for the results of Fig. 3 we used many iterations and computed the solutions in sequence of increasing resolution, using the previously computed half resolution solution as the initial estimate in each case. The iteration stopped according to (1) with $\epsilon=0.0001$ and $N_{f}=1000$. This occurred for resolutions of 2,1 , and $1 / 2^{\circ}$ after 73,210 , and 846 iterations, respectively. Higher resolution solutions terminated after 1000 iterations.

\section{4.b.2 Convergence of the minimization}

The reason for the slow convergence of the $2 \mathrm{~d}$-VAR minimization at high resolution is illustrated by Fig. 5 . This shows the results at $1 / 2^{\circ}$ resolution, beginning with a zero initial estimate, after 25,50,100, and 200 iterations. 2d-VAR initially attempts to fit the ship "obs" locally. At any iteration, if a grid point currently has a zero analysis wind increment (relative to the background) and if all its neighbors do also, then all the constraints are

perfectly satisfied and there will be no change during the next iteration. As a result the pattern of the solution slowly expands and grows during the iteration.

Many iterations were needed to show that the numerical solution is approaching a limiting (presumably true) solution. However for practical purposes it is sufficient to iterate 25 times at $2^{\circ}$, interpolate to $1^{\circ}$, iterate 25 more times, and continuing this process to the desired resolution. We call this process regridding. Regridding with only 25 iterates per resolution step provides agreement to the most accurate solution to within $1 \mathrm{~ms}^{-1}$ at resolutions of $1 / 2^{\circ}$ and better, and agreement to the converged solution for the same grid increment to 
$1 \mathrm{~ms}^{-1}$ or better at all resolutions. The latter result is illustrated by Fig. 6 , and the former may be inferred from Fig. 6 and Fig. 4.

\section{4.b.3 Sensitivity to the $\lambda$ weights}

Analyses with varying $\lambda$ weights were made using $1 / 2^{\circ}$ resolution. In these analyses (and in analyses presented in following sections), up to 1000 iterations were allowed after initial estimates were developed using the regridding process described in the previous section. In Table 2 the total number of function evaluations used is listed in the column headed $N_{f}$. This number is approximately 60 more than the number of iterations used in the final minimization.

Fig. 7 shows selected results obtained by varying some of the $\lambda$ weights by a factor of 4. The actual $\lambda$ weights used are listed in Table 2, except that in all cases $\lambda_{\mathrm{VWM}}=1$, and the background weight is effectively altered by changing all other weights in the opposite sense. Changing the "obs" weight (panel "obs', +) changes the amplitude of the response, but does not change the shape or scale of the response. In fact the solutions for different $\lambda_{\text {Conv }}$, with everything else held constant, are remarkably well correlated in the sense that one solution is nearly equal to another solution times a constant. Increasing the filtering and dynamic constraints (panel Constraints +) gives a solution of larger spatial scale, with smaller amplitude and less return flow to the right and left of the wind "obs". Decreasing the weight given the fit to the background (panel Background -) provides a generally bigger response of the same shape, but larger spatial scale, relative to the nominal case.

The half-width at half-height of the 2 d-VAR response to a single "obs" might be used to define the effective resolution of the analysis. Fig. 7 shows that this resolution is controlled by the weight given the filtering and dynamic constraints relative to the background constraint. On the other hand the amplitude of the $2 \mathrm{~d}-\mathrm{VAR}$ response can be controlled by varying $\lambda_{\text {conv }}$. For the nominal case changing some of the $\lambda_{m}$ by a factor of 4 results in a $25-30 \%$ change in amplitude or scale of the response (Table 2 and Fig. 7 ).

Fig. 8 shows selected results obtained by setting some of the weights to zero. In general, 
eliminating a constraint increases the amplitude of the analysis. Eliminating the divergence and vorticity penalty functions (panel w/o DIV, VOR) gives a solution of similar spatial scale, with larger amplitude and greater return flow to the right and left of the wind "obs". Eliminating the dynamic constraint (panel w/o DYN) results in a less elongated solution, less return flow and larger amplitude at the "obs" location. Eliminating both the divergence and vorticity penalty functions and the dynamic constraint (panel LAP only) gives a very symmetric pattern with no variation in wind direction. In this case the problem for each velocity component is effectively decoupled. In the cases without the dynamic constraint (the right column of Fig. 8), there is no dependence on the Coriolis parameter or indeed on location, and the solutions are symmetric about the line defined by the observed wind vector.

\section{4.b.4 Effect of interpolation}

In general gridded values of wind components are interpolated to the "obs" locations. In the experiments described before, the "obs" location is coincident with a grid point. In such cases the interpolation weight is one for the coincident grid point, and zero for all other grid points. Effectively, no interpolation takes place in such cases.

To examine the effect of interpolation we shifted the "obs" location to $42.25 \mathrm{~N}, 49.75 \mathrm{~W}$. Analyses were created for varying resolutions and for bilinear and bicubic interpolation. Fig. 9 shows differences with respect to the solution for $1 / 4^{\circ}$ resolution. Such differences show the combined effects of interpolation and resolution, and should be compared with Fig. 4. Note that for resolutions of $1 / 4^{\circ}$ and finer, the "obs" location is coincident with a grid point, and the solution does not depend on the interpolation procedure used. The $1 / 4^{\circ}$ solution is identical, but shifted by $1 / 4^{\circ}$ north and east with respect to the nominal case.

The linear interpolation produces a solution with larger analyzed values. This is anticipated since bilinear interpolation on a grid cell, unlike higher order interpolations, can not produce any values outside the range of the gridded values surrounding the grid cell.

In general the interpolation errors are the same size or smaller than the resolution errors. 
However at $1 / 2^{\circ}$ resolution, linear interpolation errors are noticeable, while cubic interpolation introduces errors which are small compared to the effect of resolution.

\section{4.b.5 Solutions with two observations}

A second "obs" at $45 \mathrm{~N}, 50 \mathrm{~W}$, of $27 \mathrm{~ms}^{-1}$ from $350^{\circ}$ is added to our nominal experiment. The two observations are similar to the two critical observations close to the center of the QE II storm studied by Hoffman (1982 [22]). The response of 2d-VAR to "a second observation" (panel in Fig. 10) by itself is analogous to the results of the nominal case. That is, the response is aligned with the observed wind direction, and is proportional to the observed wind speed. Adding a second observation results in a solution due to "two observations" which is qualitatively in agreement with, but weaker than, the "sum of solutions" for each observation separately. However, the sum of the individual solutions is considerably weaker than the solution for the two observations.

\section{4.c Discussion}

Clearly there is room for improvements in the minimization procedures used. In other problems of this sort good preconditioning is vital for fast convergence. This may be helpful, but all variables in the present problem are wind components and have uniform scaling. Furthermore, the discussion regarding Fig. 5 shows that the number of iterations required must grow at least linearly with the number of grid intervals required to traverse the domain. Therefore the multigrid strategy of solving the same problem with increasing grid resolution initialized from the previous solution should be generally useful. A spectral representation might also be helpful in this regard. Alternatively, but for conventional observations only, a good initial estimate at any resolution might be obtained by a Green's function approach. In this approach we would add approximations to the solution for each single "obs" to the background. For each "obs" a standard solution would be rotated to alignment with the "obs" and scaled by the difference between the "obs" and background to provide the approximate single "obs" solution. 
The results described here have some bearing on a high resolution $2 \mathrm{~d}-V A R$. With low weight given to the background and high weight given to the filtering and dynamic constraints the response of $2 \mathrm{~d}-\mathrm{VAR}$ is spread out. Any resolution with a grid spacing small enough to resolve the response will give the same result when fully converged. A high resolution analysis requires greater data density and less weight given to the filtering and dynamic constraints. With this adjustment to the $\lambda$ weights, the response of $2 \mathrm{~d}$-VAR is spatially narrower. For this reason and to resolve the details potentially present in a high density data set, smaller grid spacing is required.

\section{Comparison to OI}

As mentioned earlier, 2d-VAR can be considered a form of OI in which the background error correlations are parameterized with a small number of degrees of freedom. Here we demonstrate that with properly chosen $\lambda$ weights, $2 \mathrm{~d}-\mathrm{VAR}$ produces analyses similar to those produced by OI. It should be noted that OI uses geostrophy as a strong constraint, while $2 \mathrm{~d}$ VAR uses the time rate of change of vorticity computed from the full primitive equations (as described in $\S \mathrm{C}$ ) as a weak constraint. Thus differences might be expected in the tropics. The example shown here is therefore in the extratropics.

Fig. 11 shows the NSCAT observations in the region used for the experiments described in this section. For the purposes of comparing assimilation techniques, the unique $25 \mathrm{~km}$ NSCAT winds are thinned to $\sim 75 \mathrm{~km}$ resolution by retaining only every third WVC across track. Fig. 12 shows the background wind field used. NSCAT observation locations are marked by small black dots and the background wind speed is contoured every $2 \mathrm{~ms}^{-1}$.

The implementation of OI used, the Theater Analysis Procedures (TAP) (Nehrkorn 2000, Nehrkorn and Hoffman 1996 [41, 42]), is similar to the Navy FNMOC OI of Goerss and Phoebus (1992 [16]). Standard values of the statistics were used here, treating the scatterometer winds as ship reports with a default wind component error standard deviation $\left(s_{u}\right)$ of $2.2 \mathrm{~ms}^{-1}$. 
Fig. 13 shows an OI analysis for a region of rev 2607 at 2000 UTC 15 February 1997. For this analysis, $s_{u}$ has been increased by a factor of two to avoid overfitting the dense satellite observations. Fig. 14 shows the analysis increments (analysis-background) for the analysis in Fig. 13. The increments are significant and broaden the circulation around the cyclone as seen in the observations. Statistics for the background and analyzed wind field are found in Table 3. The rms vector analysis increment is $2.716 \mathrm{~ms}^{-1}$.

We then tried to match a $2 \mathrm{~d}-\mathrm{VAR}$ analysis to the OI solution for the same swath of data. In our search, we fixed the $2 \mathrm{~d}-\mathrm{VAR} \lambda$ weights for background and smoothness constraints $\left(\left(\lambda_{V W M}, \lambda_{L A P}, \lambda_{D I V}, \lambda_{V O R}, \lambda_{D Y N}\right)=(1,2,8,2,8)\right)$, and varied the weight given to the data $\left(\lambda_{\text {CONV }}=5,10,20\right)$.

Table 3 also shows the differences between the 2d-VAR and OI analysis presented above. A particularly good match, with $r m s$ vector wind difference of only $0.584 \mathrm{~ms}^{-1}$ was obtained using $\lambda_{C O N V}=5$.

\section{Ambiguity removal approach}

\section{6.a Definition of scatterometer "obs" functions}

For conventional ship or buoy observations $J_{\mathrm{CONV}}$ is simply the sum of squared vector differences between the "obs" and the analysis. For scatterometer data, we use two formulations. The first formulation, the method of $\mathrm{H} 84$, uses the retrieved scatterometer wind ambiguities in two "obs" functions- $J_{\mathrm{AMB}}$ and $J_{\mathrm{SPD}}$. For a single location, when the analysis is close to one of the ambiguities, the misfit measured by $J_{\mathrm{AMB}}$ approximates the squared vector differences between the ambiguity closest to the analysis and the analysis. Away from the ambiguities $J_{\mathrm{AMB}}$ approaches a constant. The variability in wind speed among ambiguities at a single location is relatively small (H84). Therefore the observed mean or $r m s$ wind speed averaged over the ambiguities at each location is representative of the scatterometer wind speed. This value is used in the definition of $J_{\mathrm{SPD}}$. The second formulation uses the observed backscatter values in $J_{\text {NRCS }}$. For a single location, the misfit measured by $J_{\text {NRCS }}$ is 
the likelihood function used to retrieve ambiguities. The likelihood function is equal to the squared normalized differences between the simulated and observed scatterometer $\sigma^{0}$ values. An optional log term may be included when the $\sigma^{0}$ variance is parameterized in terms of the analyzed $\sigma^{0}$ as explained below. Thépaut et al. (1993 [56]) used a slightly simplified form of $J_{\text {NRCS }}$.

Alternative formulations have been suggested for the loss function for scatterometer data. For example, operational use of ERS-1 and ERS-2 data at ECMWF uses the formulation of Stoffelen and Anderson (1997 [54]). Also, the variational QC of Andersson and Järvinen(1999 [1]) might be extended to scatterometer data, by treating each ambiguity as a ship report. These reports would be inconsistent at each location and the $\mathrm{QC}$ algorithm would essentially select one ambiguity at each location.

Example plots of $J_{\mathrm{NRCS}}, J_{\mathrm{AMB}}$ and the ECMWF formulation are given in Fig. 15 for a single WVC. The precise definitions of $J_{\mathrm{NRCS}}, J_{\mathrm{AMB}}$ and $J_{\mathrm{SPD}}$ are given in the following subsections. If $\sigma^{0}$ values are used directly in $2 \mathrm{~d}-\mathrm{VAR}$, the loss function due to a single WVC is the MLE (Fig. 15a). This surface shows the complexity of the highly nonlinear wind retrieval problem. Note that the center of the figure is not shaded because the values there are very large, $O\left(10^{5}\right)$, compared to those plotted in the vicinity of the minima, $O\left(10^{0}-10^{2}\right)$. The MLE has multiple minima, four in this case, which correspond to the ambiguous wind solutions. The minima are distributed in an annulus of near-minimum values at roughly the same wind speed. The four minima are not equally deep, which implies that each solution has a slightly different likelihood of being the true wind. In fact, if there were no "obs" or model function errors, only one solution would be found--the true wind.

If ambiguous winds are used in 2d-VAR, then the loss function of a single WVC is $J_{\mathrm{AMB}}$ (Fig. 15b). This approximation of $J_{\text {NRCS }}$ loses some of the structure seen in Fig. 15a, but still reflects the four wind solutions as minima and is less severely nonlinear, especially near the origin. Note that if the background wind field is close to one of the four wind solutions, the influence of other observations or meteorological constraints will be necessary to shift the analysis from near one minimum to another during the $2 \mathrm{~d}-\mathrm{VAR}$ minimization. 
For comparison, we also show the two-wind loss fuction of Stoffelen and Anderson used at ECMWF (Fig. 15c). This is the best behaved of the three functions, but is also the crudest approximation to the MLE. Only the two most likely winds are used since one of these solutions is very close to the true wind more than $90 \%$ of the time. The smooth nature of this function allows the analysis to be moved from one minima to another during the assimilation more easily than either the MLE or H84 formulation. However, this formulation may also permit an analysis solution which is not very close to either minima because of its smoothness.

\section{6.a.1 Loss function for scatterometer backscatter values}

For each backscatter "obs", the normalized departure is

$$
z_{\sigma}=\frac{\sigma^{0}{ }_{o}-\sigma^{0}{ }_{a}}{s_{\sigma}}
$$

Here

- $\sigma^{0}{ }_{o}$ is the backscatter observation.

- $\sigma^{0}{ }_{a}$ is the simulated backscatter observation.

- $s_{\sigma}$ is the standard deviation of the $\sigma^{0}$ "obs".

Theoretical estimates of the standard deviation of scatterometer measurements show that

$$
s_{\sigma}=K_{p} \sigma^{0}
$$

(Fischer 1972 [14]). Here $K_{p}$ is proportionality constant. The subscript $p$ is used because $K_{p}$ is originally defined in terms of the radar power. The JPL model for the standard deviation of the NSCAT $\sigma^{0}$ "obs" extends this model to

$$
s_{\sigma}^{2}=K_{p A}\left(\sigma^{0}\right)^{2}+K_{p B} \sigma^{0}+K_{p C}
$$

(Long et al. 1988 [36]). (For ERS-1,2 (14) is used.) When evaluating $s_{\sigma}, \sigma^{0}$ may be the observed value or the simulated value. If the simulated value is used, a logarithm term 
should be added to the loss function to be consistent with maximum likelihood estimation theory. That is

$$
J_{\mathrm{NRCS}}=\sum\left[z_{\sigma}^{2}+\ln \left(s_{\sigma}\right)\right]
$$

The term in brackets comes about from taking the logarithm of minus the normal probability function of $\sigma^{0}{ }_{a}$ given $\sigma^{0}{ }_{0}$. In practice the logarithmic term is not normally used.

A model function or forward model is used to simulate $\sigma^{0}$. As described in $\S 2$, the model function calculates $\sigma^{0}$ as a function of wind speed and direction and the geometry and polarization of the observation. The geometry is specified in terms of the pointing direction of the antenna and the incidence angle. For NSCAT the NSCAT-2 model function is preferred.

\section{6.a.2 Loss function for ambiguous scatterometer winds}

For ambiguous wind vectors the formulation of $\mathrm{H} 84$ is used. At each WVC,

$$
J_{\mathrm{AMB}}=\sum s_{\mathrm{NSCAT}}^{-2} \prod_{k=1}^{K} d_{o}^{2}\left[1-\exp \left(-d_{k}^{2} / d_{o}^{2}\right)\right],
$$

where the sum is over all WVCs, and

$$
d_{k}^{2}=\left(u_{a}-u_{k}\right)^{2}+\left(v_{a}-v_{k}\right)^{2} .
$$

Here

- $K$ is the number of ambiguities.

- $k$ is the index of the ambiguities.

- $\left(u_{k}, v_{k}\right)$ is the $k$ th wind ambiguity.

- $d_{k}$ is the magnitude of the vector difference between analysis and ambiguity $k$.

- $d_{o}$ is the wind speed scale for the "obs".

$$
d_{o}=V_{o} / \gamma
$$


- $V_{o}$ is the mean or rms wind speed for the "obs". The mean or $r m s$ is over the $K$ ambiguities,

$$
V_{o}=K^{-1} \sum_{k=1}^{K} \sqrt{\left(u_{k}\right)^{2}+\left(v_{k}\right)^{2}}, \quad \text { or } \quad V_{o}^{2}=K^{-1} \sum_{k=1}^{K}\left(u_{k}\right)^{2}+\left(v_{k}\right)^{2} .
$$

- $\gamma$ is the scaling factor for the wind speed.

- $s_{\text {NSCAT }}$ is the wind component standard deviation for the WVC.

Nominal values for $\gamma$ and $s_{\mathrm{NSCAT}}$ are 2 and $1 \mathrm{~ms}^{-1}$ respectively.

\section{6.a.3 Loss function for scatterometer wind speed}

The wind speed operator is designed to take advantage of the relatively unambiguous wind speed information in scatterometer data. In analogy to (6) we define $J_{\mathrm{SPD}}$ as,

$$
J_{\mathrm{SPD}}=\sum \frac{\left(V_{a}-V_{o}\right)^{2}}{s_{V}^{2}}
$$

Here

- $V_{a}$ is the analyzed wind speed.

- $V_{o}$ is the observed wind speed.

- $s_{V}$ is the wind speed error standard deviation.

This formulation has been used with SSM/I observations (Atlas et al. 1991, 1996 [2, 3]). The same loss function may be used for line-of-sight (LOS) wind speed in place of wind speed as long as the analyzed LOS wind speed can be calculated. This requires knowledge of the antenna pointing direction. In $\mathrm{H} 84$ a different wind speed functional was introduced. It is equivalent to (21) if $s_{V}$ is multiplied by the factor $\sqrt{1+V_{o} / V_{0}}$, where $V_{0}$ is a constant $2 \mathrm{~ms}^{-1}$. Note that this has the effect of giving more weight to low wind speed reports. 


\section{6.b Scatterometer quality control}

\section{6.b.1 Gross checks}

Gross checking is performed when the data is read from source files and prevents nonsensical or nonphysical values from being stored in $2 \mathrm{~d}$-VAR data structures. For example, NSCAT values of $\sigma^{0}>30 \mathrm{~dB}$ are eliminated. Small negative values of $\sigma^{0}$ are nonphysical but are indicative of very light winds (Pierson 1989 [46]), and in 2d-VAR are replaced with a small positive value equivalent to $-60 \mathrm{~dB}$.

\section{6.b.2 Background and analysis checks}

Background and analysis checks are done by comparing observations with the current analysis. At the start of the process, when the current analysis is the background, this procedure is a background check. Later it is a preliminary analysis check. Data failing the background check may be considered by a later (and stricter) analysis check. This allows some of the data eliminated by earlier QC back into the analysis.

As in H84, if the magnitude of the difference between wind vectors (or wind speeds) is greater than the average of the observed and analyzed wind speed, the observation fails QC. If the difference between the wind directions is $>60^{\circ}$, the observation fails QC. However, very small or zero winds are handled as a special case, so that when both observation and analysis indicate calm conditions, the previous checks are bypassed. The background and analysis checks apply to conventional data as well as to scatterometer winds. For ambiguities, only the ambiguity closest (i.e., with the smallest vector difference) to the current analysis is checked.

For NSCAT $\sigma^{0}$ data, we use a simple departure test for each $\sigma^{0}$ measurement in dB space.

$$
\sigma_{0}^{0}-\sigma_{a}^{0}>\gamma_{N S C A T}
$$

We have used $\gamma_{N S C A T}=9 d B$ for both background and analysis checks, which typically eliminates $\sim 5 \%$ of the data. 


\section{Use of ambiguous winds versus $\sigma^{0}$ observations}

When using $\sigma^{0}$ observations, 2d-VAR essentially carries out a simultaneous retrieval of NSCAT winds for all data presented to the analysis. If NSCAT ambiguous winds and $\sigma^{0}$ data represent the same geophysical information, namely ocean surface wind stress, we expect 2d-VAR to produce similar wind analyses using either data source. If this is correct, $\lambda$ for ambiguous wind and $\sigma^{0}$ data must exist which produce similar analyses. To determine such equitable $\lambda$ for ambiguous winds and $\sigma^{0}$ data, we can examine any measure of the misfit between data and analysis versus a range of $\lambda$. We say the $\lambda$ are equitable when the misfit of the analysis of the ambiguous winds is the same as the misfit of the analysis of the $\sigma^{0}$ data. This provides an example of tuning the $\lambda$ weights. Note that differences between winds-based and $\sigma^{0}$-based analyses will also arise for other reasons:

1. Quality control decisions made for $\sigma^{0}$ and wind data may result in different data being used,

2. WVCs with no ambiguities may contain valid $\sigma^{0}$ data, and

3. The ambiguous wind observation loss function, $J_{\mathrm{AMB}}$, is an approximation to the $\sigma^{0}$ data loss function $\left(J_{\mathrm{NRCS}}\right)$ (compare Figs. 15a and 15b).

The first two reasons will only effect a small fraction of the data presented to 2d-VAR while the third reason may effect all data to a small degree. With respect to the second reason, note that a single $\sigma^{0}$ value is not sufficient to infer a set of ambiguities but is sufficient to refine the background wind in 2d-VAR. With respect to the third reason, the $\sigma^{0}$ loss function $\left(J_{\mathrm{NRCS}}\right)$ will permit a wider variety of wind directions in the minimizing analysis compared to the ambiguous winds loss function $\left(J_{\mathrm{AMB}}\right)$, because of the shapes of the functions surfaces. That is, $J_{\text {NRCS }}$ has an annulus of minimum values (all wind directions) with secondary local minima embedded while the minima in $J_{\mathrm{AMB}}$ are more clearly separated.

To evaluate the analysis fit to the data, we use the $r m s$ wind speed difference between the 2d-VAR analysis interpolated to NSCAT WVCs and the first ranked (i.e., highest MLE) 
JPL ambiguity. For two North Atlantic cases, one on 19 September 1996 of Hurricane Lili in the western Atlantic and one on 27 October 1996 of a cyclone west of Ireland, analyses using ambiguous winds and $\sigma^{0}$ data were created using a range of $\lambda$. In these experiments the nominal setup includes a $1 \times 1^{\circ}$ grid over an area $20^{\circ}$ of latitude by $20^{\circ}$ longitude centered over an NSCAT data swath. The analyzed field is interpolated to observation locations by linear interpolation. The $\lambda_{i}$ for background constraints are held fixed for all analyses at

$$
\left(\lambda_{\mathrm{VWM}}, \lambda_{\mathrm{LAP}}, \lambda_{\mathrm{DN}}, \lambda_{\mathrm{VOR}}, \lambda_{\mathrm{DYN}}\right)^{T}=(1,1,4,1,16)^{T} \text {. }
$$

The minimizations were allowed to run until the gradient test was satisfied $\left(\epsilon=10^{-3}\right)$. The $\lambda$ weights used for the winds and $\sigma^{0}$ analyses are

$$
\lambda_{\mathrm{AMB}, \mathrm{NRCS}}=1 / 1024,1 / 256,1 / 64,1 / 16,1 / 4,1,4,16,64,256,1024
$$

Smaller $\lambda$ weights for scattterometer data required fewer iterations to reach convergence (typically 10-30), and larger $\lambda$ weights required more (200-600). The stronger constraint imposed by larger $\lambda$ weights $(>4)$ often caused the minimization to fail, since a new search direction could not be found to reduce the cost function any further.

Fig. 16 shows the rms analysis-NSCAT observations difference as a function of $\lambda$ weight for both ambiguous wind (w) and $\sigma^{0}(\mathrm{~s})$ analyses for the October 27 case. For very small $\lambda$, the analyses of wind and $\sigma^{0}$ data are scarcely changed and fit the wind observations equally poorly $\left(\sim 4 \mathrm{~ms}^{-1}\right)$. For large $\lambda(>4)$, the analyses of wind and $\sigma^{0}$ data fit the wind observations much more closely, and again, equally $\left(\sim 1.5 \mathrm{~ms}^{-1}\right)$. The lower limit of the $r m s$ fit is governed by our choice of $\lambda_{i}$ for the background constraints. If the background constraints were weakened (i.e., smaller $\lambda_{\mathrm{VWM}}, \lambda_{\mathrm{LAP}}, \lambda_{\mathrm{DIV}}, \lambda_{\mathrm{VOR}}$ ), the fit of the analysis to wind observations would improve. In the limit of no background constraints, the analysis would fit the observations exactly. But without any constraints on smoothness or divergence and vorticity, the solution may be unrealistic. The lower limit of $r m s$ fit is, in this case, also dependent on the robustness of the minimizer. The minimizations in analyses with $\lambda_{N R C S} \geq 1 / 4$ and $\lambda_{A M B} \geq 256$ fail to converge sufficiently because a direction of descent 
cannot be found to within machine precision. This explains why the fit to $\sigma^{0}$ does not improve for $\lambda_{N R C S} \geq 1$.

A background field, temporally interpolated to 2230 UTC October 27, 1996 from 6hourly NCEP $1000 h P a$ wind analyses, was used for the tuning experiments presented next (see Fig. 17). (The other tuning case - Hurricane Lili, October 17, 1996 - produced similar results and will not be shown.) To illustrate that ambiguous winds and $\sigma^{0}$ data represent the same information, Fig. 18 shows analyses and increments for $\lambda_{A M B}=4$ and $\lambda_{N R C S}=1 / 4$. The upper panels show 2d-VAR analyses and the lower panels show analysis increments times ten. The analyses are very similar in pattern and magnitude. While the analysis domain rms vector magnitude is $3.144 \mathrm{~ms}^{-1}$ for the winds-based analysis increments and $2.198 \mathrm{~ms}^{-1}$ for the $\sigma^{0}$-based analysis increments, it is $1.856 \mathrm{~ms}^{-1}$ for the difference of the two analyses. Differences between the analyses are subtle. The center of the low is displaced very slightly to the east in the ambiguous winds analysis relative to the background and the $\sigma^{0}$ analysis. Also, in the region south of Ireland, winds from the southwest in the background are changed to more southerly winds in the ambiguous winds analysis (easily seen in the increments, lower panels). This change produces a circulation pattern around the cyclone which is slightly less circular than in the $\sigma^{0}$ analysis. This is very likely due the the difference in the observation loss functions. $J_{\mathrm{AMB}}$ draws the solution toward one of the wind ambiguities, generally not permitting a solution to fall between minima. In contrast, $J_{\text {NRCS }}$ tends to allow any solution within the annulus of minimum values, with only a minor preference for the retrieved solutions (i.e., for the local minima within the annulus). Apart from these minor differences, it is clear that wind and $\sigma^{0}$ data, while handled very differently by $2 \mathrm{dVAR}$, produce nearly the same result given an appropriate choice of $\lambda_{A M B}$ and $\lambda_{N R C S}$.

\section{Dual ambiguity processing}

While more than two winds are often retrieved from a group of colocated $\sigma^{0}$ measurements, one of the two ambiguities with the highest MLE values is very likely (>90\%) to be closest 
to the true wind (JPL 1997 [32]). This implies that $<10 \%$ of the third and fourth ranked ambiguities are closest to the true wind. This information can be used to QC the data given to $2 \mathrm{~d}-\mathrm{VAR}$, and improve ambiguity removal.

We observed that using all available ambiguities in $2 \mathrm{~d}-\mathrm{VAR}$, or equivalently using the $\sigma^{0}$ values, leads to patches of poor ambiguity selection. This is especially true where there are four ambiguities and the direction of the the third or fourth ambiguity is close to the direction of the background wind field. Using "dual ambiguities" (i.e., the two ambiguities with the highest MLE values) narrows the choice to one of the two most likely possibilities. This allows for larger $\left(>45^{\circ}\right)$ wind direction corrections to the background field. Using dual ambiguities only, however, naturally limits ambiguity selection to the first or second ranked ambiguity since the analysis will generally be drawn toward one or the other.

To allow for selection from all ambiguities, we have developed a two stage analysis procedure which blends the use of dual and all ambiguities. In the first stage, we use dual ambiguities for 50 iterations of the minimizer to draw the analysis toward one of the two most likely observed winds. In the second stage, we include all ambiguities and continue to minimize until the convergence criteria are met. An alternative is to switch to $\sigma^{0}$ values after the first stage. The second stage allows for readjustment of the analysis in the few locations where the third or fourth ranked ambiguity is closest to the true wind.

During the first stage, we only use dual ambiguities which pass dual ambiguity quality control or "dual QC". Dual QC is based on a conceptual model of dual ambiguities. Nominally, we expect the wind directions of dual ambiguities to be opposed by $\sim 1800^{\circ}$ If not, the retrieved winds are suspect and are not included in the first (dual ambiguities) stage. Specifically, retrieved winds fail dual QC and are not used in the first minimization if the directions of the two most likely ambiguities are within $3 \pi / 4$ of each other. Dual QC typically eliminates $\sim 20 \%$ of WVC's from the first minimization. 


\section{8.a Dual QC ambiguity removal examples}

One measure of the performance of ambiguity removal with $2 \mathrm{~d}$-VAR is a direct comparison with another successful ambiguity removal scheme. Here we compare our results with the NWP-initialized or "nudged" median filter used by JPL for ambiguity removal, and with ambiguities chosen to be closest to the NWP analysis used as the background. 2d-VAR winds and median filter winds agree in a large proportion of the WVCs, typically $95 \%$. Detailed statistical comparisons for the whole NSCAT mission are given by Henderson et al. (2001 [21]).

In cases of disagreement both sets of winds are still horizontally coherent. In our synoptic evaluation, we find $2 \mathrm{~d}-\mathrm{VAR}$ winds tend to be more meteorologically reasonable. Recall that both methods are nonlinear and have multiple solutions. Because $2 \mathrm{~d}-\mathrm{VAR}$ requires dynamic consistency and the median filter does not, solutions of the median filter are not necessarily solutions of 2d-VAR. For example, two blocks of winds with opposite directions can satisfy the median filter, but not 2d-VAR.

As a test of our dual-QC procedure we initially processed 234 orbits of NSCAT data based on the NSCAT -1 model function during the period 15 October-5 November 1996. JPL and 2d-VAR ambiguity selection differences are predominately at lower wind speeds (Table 4). To further assess the performance of 2d-VAR, we use GOES- 8 imagery to locate mesoscale meteorological features in the North Atlantic, for the subset of 29 orbits passing over the North Atlantic when the sun elevation angle was sufficient to make use of the GOES visible channel (Grassotti et al. 1999 [20]). We examined 11 North Atlantic scenes where JPL and 2d-VAR ambiguity selection differed. From the 11 scenes, 29 subregions were examined in detail. For brevity, we present one of the subregions below and then summarize the results of all 29.

\section{8.a.1 Case of 26 October 1996}

Fig. 19 shows GOES-8 IR brightness temperature over the North Atlantic at 1315 UTC 26 October 1996. NSCAT data locations are marked by the fine black dots, and WVC's 
where JPL and 2d-VAR-selected ambiguities differ are marked by heavy blue dots. The scatterometer crossed 30N at 1314 UTC. Differences between JPL and 2d-VAR-selected ambiguities occur in small patches near $(35 \mathrm{~W}, 29 \mathrm{~N}),(37 \mathrm{~W}, 41 \mathrm{~N}),(30 \mathrm{~W}, 45 \mathrm{~N})$, and $(32 \mathrm{~W}, 57 \mathrm{~N})$ in Fig. 19. In region A, satellite imagery shows that the scatterometer passed over a mature cyclone at $36 \mathrm{~W}, 41 \mathrm{~N}$.

Fig. 20 shows ECMWF (thin red barbs) and 2d-VAR (thick black barbs) analyses in region A over an image of GOES-8 IR brightness temperature. NSCAT WVC locations are marked by small black circles, and points where JPL and 2d-VAR ambiguity selection differ are marked by larger blue circles with white centers. It is interesting to note several effects of the NSCAT data on 2d-VAR surface wind analysis. The circulation center in 2d-VAR analysis is moved north and east of the center in the ECMWF analysis. The placement in the $2 \mathrm{~d}-\mathrm{VAR}$ analysis is in better agreement with the satellite image. Also, the wind speeds around the cyclone are higher (roughly doubled) in the $2 \mathrm{~d}-\mathrm{VAR}$ analysis than in the ECMWF analysis.

All JPL ambiguities in subregion A1 of Fig. 20 are plotted in Fig. 21. The ambiguous winds are consistent with a cyclone centered at 36W, 41N. Fig. 22 shows JPL and 2d-VAR selected ambiguities for subregion A1. A physically unlikely east-west wind shift exists in the JPL ambiguites in the northwest quadrant of the storm $(37 \mathrm{~W}, 41.5 \mathrm{~N})$. The satellite imagery is more consistent with the circular wind flow around the cyclone center evident in Fig. 22b, which shows $2 \mathrm{~d}$-VAR selected ambiguities. Note that $2 \mathrm{~d}-\mathrm{VAR}$ positions the center of circulation $\geq 50 \mathrm{~km}$ south of the wind shift in the JPL selected ambiguities.

\section{8.a.2 Summary of All Cases with Colocated GOES Imagery}

The subregion presented in $\S 8 . \mathrm{a} .1$ is one of 29 cases where $2 \mathrm{~d}-\mathrm{VAR}$ and JPL ambiguity selection differ that were examined for the three week period. These cases include cloudy and clear scenes, tropics and mid-latitudes, and coastal and open water regions. The results from each subregion are categorized by the differences between the JPL and 2d-VAR selected ambiguities. Using GOES-8 imagery and general knowledge of the local wind field 
from ECMWF analyses, 2d-VAR selected ambiguities are determined to be either 1) clearly improved over JPL selected ambiguities, 2) different from JPL but equally plausible, or 3) clearly worse than JPL selected ambiguities. For reference, 2d-VAR selected ambiguities were determined to be a clear improvement over JPL selected ambiguities for the subregion presented in $\S 8$.a.1. For the 29 subregions we examined,

- 10 subregions are clearly improved over JPL selected ambiguities

- 16 subregions are different from JPL but equally plausible

- 3 subregions are clearly worse than JPL selected ambiguities

Cases classified as "equally plausible" often occur when the evidence from the imagery is not decisive. For example, sometimes a front is aligned with a cloud feature in both 2d-VAR and JPL selections but displaced one or two WVCs in the perpendicular direction. In other "equally plausible" cases all ambiguities are inconsistent with the imagery.

\section{Concluding remarks}

2d-VAR provides an alternative method of ambiguity removal for NSCAT data. In this paper we describe both 2d-VAR and its application to NSCAT data. Experiments with a single ship observation illustrate the numerical behavior and sensitivity of $2 \mathrm{~d}$-VAR. Note that single observation experiments with $2 \mathrm{~d}$-VAR when normalized to have a unit response at the "obs" location, are the background error correlations with the single "obs". (This is a general result: Thépaut et al. (1993 [56]) essentially showed this for a more complicated situation.) In experiments based on no tuning other than the subjective tuning described by H84, the response of $2 \mathrm{~d}-\mathrm{VAR}$ to a single ship observation is very similar to the response of OI analyses making use of models of forecast error statistics used operationally by FNMOC (Goerss and Phoebus 1992 [16]). Therefore the subjectively tuned 2d-VAR approximates OI. Idealized OI experiments with a swath of unique NSCAT winds demonstrate the close relationship between 2d-VAR and OI. 2d-VAR analyses can be used to select ambiguities by choosing 
the ambiguous wind closest to the analyzed wind field at each WVC location. Comparisons between the use of ambiguities and backscatter in $2 \mathrm{~d}$-VAR show the near equivalence of these two data for this purpose and also demonstrate tuning the $\lambda$ weights. Whether using ambiguities or backscatter, the objective function is highly nonlinear-multiple solutions are possible. The analysis tends to settle on the ambiguity closest to the background field. When the background quality is not sufficient, the fraction of third and fourth ambiguities chosen is larger than expected: NSCAT and buoy colocations show that more than $90 \%$ of selected ambiguities should be the first or second ambiguity. This leads to the concept of "dual-ambiguities" quality control (dual-QC).

Ambiguity removal using $2 \mathrm{~d}-\mathrm{VAR}$ shows promise and may have advantages over median filter techniques. While JPL and 2d-VAR ambiguity selection differ for only $\sim 5 \%$ of WVC's, the differences tend to occur in patches. Patches of poorly selected winds can be more harmful to analysis and forecasting systems than scattered errors if not properly quality controlled. In $\sim 30 \%$ of the cases examined, 2d-VAR selected ambiguities are a clear improvement over JPL selected ambiguities. In $\sim 60 \%$ of these cases, there was either insufficient information to determine which choice was more likely or a reasonable solution was not present in the ambiguities. In the remaining $\sim 10 \%$ of these cases, the JPL selected ambiguities are preferred. In a companion article (Henderson et al. 2001 [21]), we process the entire 9month mission using the latest version of the data from JPL based on the NSCAT-2 model function.

Further work could be done to investigate the use of the scatterometer backscatter measurements $\left(\sigma^{0}\right)$ directly for ambiguity removal. This may prove most useful in cases when a suitable solution does not seem to exist in the retrieved winds. Many of the principles learned here are applicable to NASA's current Seawinds scatterometer on the QuikSCAT satellite. In addition there are other factors which we have studied in other contexts which impact ambiguity removal. Positional errors in the background field can have a substantial effect on ambiguity selection. Henderson et al. (2001 [21]) show the effect of using a very poor background. Smaller positional errors might be handled within 2d-VAR using our 
method for feature calibration and alignment (Hoffman et al. 1995, Hoffman and Grassotti 1996, Grassotti et al. 1999 [25, 24, 19]). Rain effects seem to be important for QuikSCAT (Jones et al. 2000, Mears et al. $2000[31,37]$ ). However for NSCAT, limited experiments with rain flagging show small impacts on 2d-VAR results. Grassotti et al. (1999 [20]) developed a rain flag for NSCAT data based on geostationary visible-infrared imagery. That study was a demonstration restricted to three weeks of data in the fall of 1996 in the North Atlantic. For most cases studied by Grassotti et al., the effect of removing flagged WVC on ambiguity removal using 2d-VAR was small. Still, occasionally, large differences can result from eliminating just one WVC.

\section{Acknowledgments}

Data used in the research reported here were provided by the Jet Propulsion Laboratory (JPL) Physical Oceanography Distributed Active Archive Center (PO.DAAC), the National Center for Environmental Prediction (NCEP) and the European Centre for Medium-Range Weather Forecasts (ECMWF). This research was supported by the NSCAT and SeaWinds NASA scatterometer projects.

\section{A Representation of the wind fields}

For the purpose of minimization, we consider $J$ to be a function of $\boldsymbol{X}$, which is known as the control variable. We must be able to map the control variables to a complete gridded wind field. That is

$$
V_{a}=\mathcal{M}(X)
$$

Here

- $V_{a}$ is the gridded wind analysis.

- $\mathcal{M}$ is the mapping operator from control variables to gridded winds. 
- $X$ is the control variable vector.

Nominally $V_{a}$ is the $10 m$ neutral stability wind field. All observed winds should be translated into a $10 \mathrm{~m}$ neutral stability wind. If only the height of the observation is known, then the neutral logarithmic wind profile may be used to adjust the wind observation to $10 \mathrm{~m}$. The control variables might contain grid point wind components, spectral coefficients of analysis increments, or some other representation of the wind fields. In one sense $X$ must completely describe the wind field, however any constant auxillary information may be used by $\mathcal{M}$.

The grid is specified in terms of longitude and latitude denoted for convenience by the variables $x$ and $y$, respectively. The usual setup is to evaluate all the functionals over a rectangular region in $x$ and $y$. However the data acceptance window for evaluating the $J_{o}$ terms and the integration domain for evaluating the $J_{b}$ terms may be specified individually. (In fact one could specify these domains individually for each component of these cost functions.) To begin, we define the integration domain:

For both $x$ and $y$ there is a starting location, an increment $\delta$, and a number of grid boxes $n$. These grid boxes are numbered from 1 to $n$ and the corresponding grid points are numbered from 0 to $n$,

Grid box
Grid point

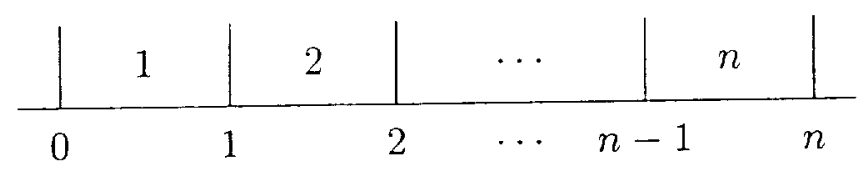

To evaluate some of the finite difference operators and to allow higher order horizontal interpolation, a boundary zone one grid box wide is added to the grid. The grid point indices therefore run from -1 to $n+1$, and the boundary grid boxes are numbered 0 and $n+1$. Therefore

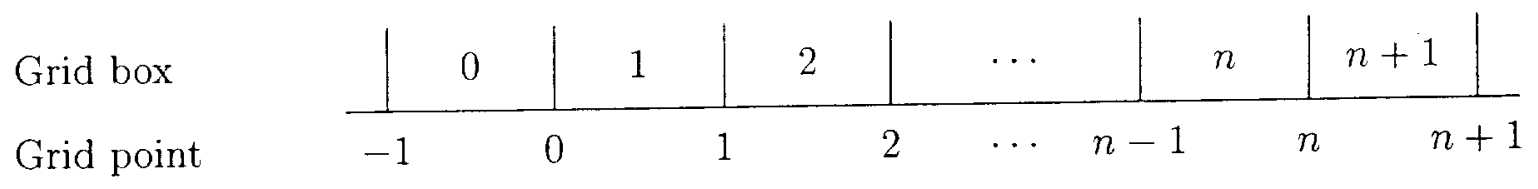

Grid points 0 and $n$ at the integration boundary may be either active or passive, i.e. allowed to vary during the minimization or held fixed. If they are held fixed they are part of the boundary. In the experiments reported here they are active and part of the control vector. 
The domain may be periodic in the $x$ direction. In this case grid points $i$ and $i+2 \pi / \delta$ are the same. Our implementation does not allow pole points.

With the grid specified, we now describe the operator $\mathcal{M}$. The fixed points of the grid are held constant or reset to their fixed values. All other values are copied from locations in $X$ to locations in $V_{a}$. Therefore $X$ may be taken to be the concatenation of portions of the gridded wind component arrays which have been reshaped into vectors. The relevant portions are free to vary and do not extend beyond one period in the $x$ direction. If the grid is periodic some values may be copied twice.

\section{B Horizontal interpolation}

The grid point values of the $(u, v)$ wind components are interpolated bilinearly to the "obs" locations. (That is, $u$ and $v$ are separately interpolated first in longitude and then in latitude.) In some experiments, a bicubic interpolator is used. Since the interpolated value is a linear function of the data values at the grid points, we simplify the adjoint calculation by formulating the interpolation as a weighted sum of grid point values. The key point is that these weights are constants depending only on the "obs" location. Interpolating in one dimension and then in the other, shows the weight for a grid value is the product of the weights for interpolating in each single dimension. In a single dimension, the weights are most easily determined from the Lagrange multiplier representation of the colocating polynomial,

$$
f(x)=\sum_{j}\left[\prod_{i \neq j} \frac{x-x_{i}}{x_{j}-x_{i}}\right] f_{j},
$$

where $f_{j}$ are the data at locations $x_{j}$. The sum and product in (24) are from 1 to $N+1$, where $N$ is the order of the polynomial. Cubic interpolation for example requires four data points. When $x$ is equal to one of the $x_{j}$, the weights in (24) reduce to one for $j$ and zero otherwise. The bicubic interpolation actually uses a 12 point mask, following Ritchie et al. (1995, Fig. 2 [48]). 


\section{Calculation of the background constraints}

The background constraints are all formulated as

$$
J_{m}=\frac{1}{S_{m}} \int_{A}\left(\mathcal{R}_{m}\left(\boldsymbol{V}_{a}\right)-\mathcal{R}_{m}\left(\boldsymbol{V}_{b}\right)\right)^{2} d A
$$

That is, each background constraint term, $J_{m}$, listed in Table 1 may be written as the scaled integral of the squared difference of some operator applied to the analysis field and to the background field.

Below, several of the operators $\mathcal{R}_{m}$ are put in the form,

$$
\mathcal{R}=\nabla \cdot Q
$$

In numerical experiments, we found that this version of the Laplacian leads to an instability in the minimization. Instead the Laplacian is calculated at each grid point and then $J_{v w m}$ and $J_{\text {LAP }}$ are calculated in the same way, by averaging four adjacent grid point values to estimate the value at the center of the grid box. (See the discussion in $\S$ C.2.)

The filtering constraints are based on minimizing the vorticity, divergence and Laplacian of the analysis increments. We call these constraints filtering because they tend to eliminate small spatial scales. The dynamic constraint is based on minimizing the analysis increments of the time rate of change of vorticity. An second similar dynamic constraint could easily be formulated in terms of divergence, but would require introducing surface pressure into the problem. The background constraints and scales are specified in terms of $S_{m}, \mathcal{R}_{m}$ and $Q_{m}$ as follows:

\begin{tabular}{|c|c|c|c|}
\hline$m$ & $S_{m}$ & $\mathcal{R}_{m}$ & $Q_{m}$ \\
\hline VWM & $T^{-2} L^{4}$ & $\boldsymbol{V}$ & - \\
LAP & $T^{-2}$ & $\left(\nabla^{2} u, \nabla^{2} v\right)$ & $\boldsymbol{V}$ \\
DIV & $T^{-2} L^{2}$ & $\nabla \cdot \boldsymbol{V}$ & $k \times \boldsymbol{V}$ \\
VOR & $T^{-2} L^{2}$ & $-\nabla \cdot k \times \boldsymbol{V}$ & $-(\zeta+f) \boldsymbol{V}-\boldsymbol{k} \times \boldsymbol{F}$ \\
DYN & $T^{-4} L^{2}$ & $\partial \zeta / \partial t$ & $-(\zeta+$ \\
\hline
\end{tabular}


Here

- $f$ is the Coriolis parameter.

- $\boldsymbol{F}$ is the surface friction term in the momentum equation.

The definitions of the $Q_{m}$ for $J_{\mathrm{DIV}}$ and $J_{\mathrm{VOR}}$ follow immediately from the definitions of the $\mathcal{R}_{m}$ and the vector identities. To compute the dynamic constraint and to determine the $Q_{\text {DYN }}$, we start with the equations from Bourke et al. (1977 [6]) for the time rate of change of vorticity. We then eliminate horizontal (but not surface) friction and vertical advection terms. Horizontal friction is negligible at synoptic scales. Formally the vertical velocity in sigma coordinates is zero at the surface.

\section{C.1 Finite differencing}

Simple finite difference forms are used. If the components of $Q$ are $(p, q)$, then in spherical geometry,

$$
\nabla \cdot Q \approx p_{x}+q_{y}-\frac{\tan \phi}{a} q
$$

and

$$
\nabla q=\left(q_{x}, q_{y}\right)
$$

where for any $p$ and $q$,

$$
p_{x, i j}=\frac{p_{i+1, j}-p_{i-1, j}}{2 a \cos y_{j} \delta x}, \quad q_{y, i j}=\frac{q_{i, j+1}-q_{i, j-1}}{2 a \delta y} .
$$

These finite difference forms are applied directly at a grid point to evaluate $\zeta$. However, the $\mathcal{R}$ are evaluated at the center of the grid box. Instead of applying (29) directly in (27) at the grid points and then averaging, we apply (27) at the center of the grid box. We evaluate $\overline{p_{x}}$ by first averaging in $y$ and then differencing in $x$. The overbar indicates a value at the center of the gridbox. Similarly, we evaluate $\overline{q_{y}}$ by first averaging in $x$ and then differencing in $y$. For $\vec{q}$, we again take the average of the corner grid point values. 


\section{C.2 Evaluation of integrals}

All integrals are put in the form of (25). For $J_{\mathrm{vwm}}$ and $J_{\text {lap }}$ there are two integrals of this form--one for each wind component.

For all $J_{m}$ we discretize the integral as

$$
J_{m}=\sum_{i, j}\left(\Delta \tilde{R}_{i j}\right)^{2} A_{i j}
$$

where

- $\Delta \tilde{R}_{i j}$ is the estimate of $\mathcal{R}_{m}\left(\boldsymbol{V}_{a}\right)-\mathcal{R}_{m}\left(\boldsymbol{V}_{b}\right)$ at the middle of grid box $i j$.

- $A_{i j}$ is the area of grid box $i j$.

As mentioned for $J_{\mathrm{VWM}}$ and $J_{\mathrm{LAP}}$, the $\tilde{R}_{m}$ are estimated as the average of the four corner grid point values. For the other constraints, the $\tilde{R}_{m}$ are estimated as described in $\S$ C.1.

\section{C.3 The gradient calculation}

Simple finite difference formula are used because $J$ is ad hoc and the $\lambda$ are adjustable. Thus we may easily subsume the approximation errors introduced by the finite difference and integration formula into the assumption that minimizing $J$ provides the optimal analysis. In other words, we identify the best analysis as the minimizer of the discrete formulation of $J$. These considerations suggest choosing simple averaging and difference forms. However, it is vital that once the finite difference version of $J$ is chosen, the gradient of $J$ be calculated as exactly and precisely as possible.

The calculation of $J_{b}$ and its gradient may be made efficient, both in terms of calculations and memory, as follows. Since both the analysis and background fields are gridded, it is possible to evaluate all of the constraints by sweeping from south to north in latitude. In addition the total background function may be considered to be the weighted sum of many partial cost functions, one for each constraint and each grid box. Since the weights are fixed and known a priori, it is possible to accumulate the contributions of these partial 
cost functions to the sensitivities of the total background function in the same sweep over latitude. 


\section{References}

[1] E. Andersson and H. Järvinen. Variational quality control. Quart. J. Roy. Meteor. Soc., 125(554):697-722, Jan. (Part B) 1999.

[2] R. Atlas, S. C. Bloom, R. N. Hoffman, J. V. Ardizzone, and G. Brin. Space-based surface wind vectors to aid understanding of air-sea interactions. Trans. Amer. Geophys. Union, $72: 201-208,1991$.

[3] R. Atlas, R. N. Hoffman, S. C. Bloom, J. C. Jusem, and J. Ardizzone. A multiyear global surface wind velocity data set using SSM/I wind observations. Bull. Amer. Meteor. Soc., $77(5): 869-882$, May 1996.

[4] F. Badran, S. Thiria, and M. Crepon. Wind ambiguity removal by the use of neural network techniques. J. Geophys. Res., 96(C11):20521-20530, 1991.

[5] D. Boggs. The Seasat scatterometer model function: The genesis of SASS I. Technical report, Jet Propulsion Laboratory, Pasadena, CA, 1981. [NTIS PD 622-230].

[6] W. Bourke, B. McAvaney, K. Puri, and R. Thurling. Global modeling of atmospheric flow by spectral methods. In J. Chang, editor, General Circulation Models of the Atmosphere, volume 17 of Methods in Computational Physics, pages 267-324. Academic, New York, 1977.

[7] R. A. Brown. On satellite scatterometer capabilities in air-sea interaction. J. Geophys. Res., 91(C2):2221-2232, 1986. Special Section: Frontiers of Remote Sensing of the Oceans and Troposphere from Air and Space Platforms.

[8] C.-Y. Chi and F. K. Li. A comparative study of several wind estimation algorithms for spaceborne scatterometers. IEEE Trans. Geosci. Remote Sens., 26:115-121, 1988.

[9] K. Conradsen, L. B. Nielsen, and L. P. Prahm. Review of Weibull statistics for estimation of wind speed distributions. J. Climate Appl. Meteor., 23(8):1173-1183, Aug. 1984. 
[10] J. C. W. de Vries and A. C. M. Stoffelen. 2D variational ambiguity removal. Technical Report 226, Royal Netherlands Meteorological Institute (KNMI), De Bilt, The Netherlands, 2000.

[11] D. P. Dee and A. M. da Silva. Data assimilation in the presence of forecast bias. Quart. J. Roy. Meteor. Soc., 124(545):269-295, Jan. (Part A) 1998.

[12] D. P. Dee, G. Gaspari, C. Redder, L. Rukhovets, and A. M. da Silva. Maximumlikelihood estimation of forecast and observation error covariance parameters. Part II: Applications. Mon. Wea. Rev., 1998. Submitted.

[13] J. Figa and A. Stoffelen. On the assimilation of Ku-band scatterometer winds for weather analysis and forecasting. IEEE Trans. Geosci. Remote Sens., 38(4):1893-1902, July 2000.

[14] R. E. Fischer. Standard deviation of scatterometer measurements from space. IEEE Trans. Geosci. Electron., GE-10:106-113, 1972.

[15] M. H. Freilich and R. S. Dunbar. The accuracy of the NSCAT 1 vector winds: Comparisons with National Data Buoy Center buoys. J. Geophys. Res., 104(C5):11231-11246, 15 May 1999.

[16] J. S. Goerss and P. A. Phoebus. The Navy's operational atmospheric analysis. Wea. Forecasting, 7:232-249, 1992.

[17] J. S. Goerss and P. A. Phoebus. The multivariate optimum interpolation analysis of meteorological data at the Fleet Numerical Oceanography Center. FR 7531-92-9413, Navy Research Laboratory, Monterey CA, Apr. 1993.

[18] A. E. Gonzales and D. G. Long. An assessment of NSCAT ambiguity removal. $J$. Geophys. Res., 104(C5):11449-11457, 15 May 1999. 
[19] C. Grassotti, R. N. Hoffman, and H. Iskenderian. Fusion of ground-based radar and satellite-based rainfall data using feature calibration and alignment. J. Appl. Meteor., 38(6):677-695, June 1999.

[20] C. Grassotti, S. M. Leidner, J.-F. Louis, and R. N. Hoffman. Development and application of a visible-infrared rain flag for scatterometer data. J. Appl. Meteor., 38(6):665676, June 1999.

[21] J. M. Henderson, R. N. Hoffman, and S. M. Leidner. A comparison of two ambiguity removal methods applied to NSCAT: a two-dimensional variational analysis method versus a median filter. J. Geophys. Res., 2001. In preparation.

[22] R. Hoffman. SASS wind ambiguity removal by direct minimization. Mon. Wea. Rev., 110:434-445, 1982.

[23] R. N. Hoffman. SASS wind ambiguity removal by direct minimization. II: Use of smoothness and dynamical constraints. Mon. Wea. Rev., 112:1829-1852, 1984.

[24] R. N. Hoffman and C. Grassotti. A technique for assimilating SSM/I observations of marine atmospheric storms. J. Appl. Meteor, 35(8):1177-1188, Aug. 1996.

[25] R. N. Hoffman, Z. Liu, J.-F. Louis, and C. Grassotti. Distortion representation of forecast errors. Mon. Wea. Rev., 123(9):2758-2770, Sept. 1995.

[26] R. N. Hoffman, J.-F. Louis, and T. Nehrkorn. A simplified view of adjoint calculations in the discrete case. Technical Memorandum 184, ECMWF, Reading, U.K., 1992.

[27] Z. Janjić. The step-mountain Eta coordinate model: Further developments of the convection, viscous sublayer, and turbulence closure schemes. Mon. Wea. Rev., 122(5):927$945,1994$.

[28] C. S. Jones, D. M. Legler, and J. J. O'Brien. Variability of surface fluxes over the Indian Ocean: 1960-1989. The Global Atmosphere and Ocean System, 3:249-272, 1995. 
[29] W. L. Jones, V. J. Cardone, W. J. Pierson, J. Zec, L. P. Rice, A. Cox, and W. B. Sylvester. NSCAT high-resolution surface wind measurements in Typhoon Violet. $J$. Geophys. Res., 104(C5):11247-11259, 15 May 1999. Correction in number C8, page 18,469 .

[30] W. L. Jones, L. C. Schroeder, and J. L. Mitchell. Aircraft measurements of the microwave scattering signature of the ocean. IEEE J. Oc. Eng., OE-2:52-61, 1977.

[31] W. L. Jones, M. Susanj, J. Zec, and J.-D. Park. Validation of QuikScat radiometric estimates of rain rate. In International Geoscience and Remote Sensing Symposium (IGARSS), Honolulu, HI, 24-28 July 2000. IEEE, New York.

[32] JPL. NASA scatterometer science data product user's manual. Version 1.1, Jet Propulsion Laboratory, Pasadena, CA, Apr. 1997. [JPL D-12985].

[33] D. M. Legler, I. M. Navon, and J. J. O'Brien. Objective analysis of pseudostress over the Indian Ocean using a direct-minimization approach. Mon. Wea. Rev., 117:709-720, 1989.

[34] D. C. Liu and J. Nocedal. On the limited memory method for large scale optimization. Mathematical Programming B, 45(3):503-528, 1989.

[35] D. G. Long. Wind field model-based estimation of Seasat scatterometer winds. $J$. Geophys. Res., 98(C8):14651-14668, 1993.

[36] D. G. Long, C.-Y. Chi, and F. K. Li. The desing of an onboard digital Doppler processor for a spaceborne scatterometer. IEEE Trans. Geosci. Remote Sens., 26:869-878, 1988.

[37] C. Mears, D. Smith, and F. Wentz. Detecting rain with QuikSCAT. In International Geoscience and Remote Sensing Symposium (IGARSS), Honolulu, HI, 24-28 July 2000. IEEE, New York. 
[38] S. D. Meyers, C. S. Jones, D. M. Legler, K. F. Miles, and J. J. O'Brien. The sensitivity to parametric variation in direct minimization techniques. Mon. Wea. Rev., 122(7):16321639, July 1994.

[39] F. M. Naderi, M. H. Freilich, and D. G. Long. Spaceborne radar measurement of wind velocity over the ocean-an overview of the NSCAT scatterometer system. Proc. IEEE, $79: 850-866,1991$.

[40] I. M. Navon and D. M. Legler. Conjugate-gradient methods for large-scale minimization in meteorology. Mon. Wea. Rev., 115:1479-1502, 1987.

[41] T. Nehrkorn. Analysis and quality control of profiler data using optimum interpolation. J. Atmos. Ocean. Technol., 17(5):651-655, May 2000.

[42] T. Nehrkorn and R. N. Hoffman. Development of a small-scale, relocatable optimum interpolation data analysis system. In 11 th Conference on Numerical Weather Prediction, pages 91-93, Norfolk, Virginia, 19-23 Aug. 1996. American Meteorological Society, Boston, MA.

[43] D. Offiler. Wind retrieval and ambiguity removal. EPIPVS Project Note 9, U. K. Met. Office, U. K. Met. Office, Bracknell, UK, 17 Dec. 1992.

[44] D. Offiler. The calibration of ERS-1 satellite scatterometer winds. J. Atmos. Ocean. Technol., 11(4):1002-1017, Aug. 1994.

[45] P. J. Pegion, M. A. Bourassa, D. M. Legler, and J. J. O'Brien. Objectively derived daily "winds" from satellite scatterometer data. Mon. Wea. Rev., 128(9):3150-3168, Sept. 2000 .

[46] W. J. Pierson, Jr. Probabilities and statistics for backscatter estimates obtained by a scatterometer. J. Geophys. Res., 94(C7):9743-9759, 15 July 1989.

[47] J. C. Price. The nature of multiple solutions for surface wind speed over the oceans from scatterometer measurements. Remote Sens. Environ., 5:47-54, 1976. 
[48] H. Ritchie, C. Temperton, A. Simmons, M. Hortal, T. Davies, D. Dent, and M. Hamrud. Implementation of the semi-Lagrangian method in a high-resolution version of the ECMWF forecast model. Mon. Wea. Rev., 123(2):489-514, Feb. 1995.

[49] R. Romeiser, W. Alpers, and V. Wismann. An improved composite surface model for the radar backscattering cross section of the ocean surface. 1. Theory of the model and optimization/validation by scatterometer data. J. Geophys. Res., 102(C11):2523725250, 15 Nov. 1997.

[50] R. Romeiser, W. Alpers, and V. Wismann. An improved composite surface model for the radar backscattering cross section of the ocean surface. 2. Model response to surface roughness variations and the radar imaging of underwater bottom. J. Geophys. Res., 102(C11):25251-25267, 15 Nov. 1997.

[51] L. C. Schroeder, W. L. Grantham, E. M. Bracalente, C. L. Britt, K. S. Shanmugam, F. J. Wentz, D. P. Wylie, and B. B. Hinton. Removal of ambiguous wind directions for a Ku-band wind scatterometer using three different azimuth angles. IEEE Trans. Geosci. Remote Sens., GE-23:91-100, 1985.

[52] H. Schultz. A circular median filter approach for resolving directional ambiguities in wind fields retrieved from spaceborne scatterometer data. J. Geophys. Res., 95(C4):5291-5304, 1990. Errata in number C6, page 9783.

[53] S. J. Shaffer, R. S. Dunbar, S. V. Hsiao, and D. G. Long. A median-filter-based ambiguity removal algorithm for NSCAT. IEEE Trans. Geosci. Remote Sens., 29:167-174, 1991

[54] A. Stoffelen and D. Anderson. Ambiguity removal and assimilation of scatterometer data. Quart. J. Roy. Meteor. Soc., 123(538):491-518, Jan. (Part B) 1997.

[55] A. Stoffelen and D. Anderson. Scatterometer data interpretation: Estimation and validation of the transfer function CMOD4. J. Geophys. Res., 102(C3):5767-5780, 15 Mar. 1997. 
[56] J.-N. Thépaut, R. N. Hoffman, and P. Courtier. Interactions of dynamics and observations in a four-dimensional variational assimilation. Mon. Wea. Rev., 121:3393-3414, 1993.

[57] J.-N. Thépaut, D. Vasiljevic, P. Courtier, and J. Pailleux. Variational assimilation of conventional meteorological observations with a multilevel primitive-equation model. Quart. J. Roy. Meteor. Soc., 119:153-186, 1993.

[58] G. Wahba and J. Wendelberger. Some new mathematical methods for variational objective analysis using splines and cross validation. Mon. Wea. Rev., 108:1122-1143, 1980.

[59] F. J. Wentz. A simplified wind vector algorithm for satellite scatterometers. J. Atmos. Ocean. Technol., 8:697-704, 1991.

[60] F. J. Wentz, S. Peteherych, and L. A. Thomas. A model function for ocean radar cross sections at 14.6 GHz. J. Geophys. Res., 89(C3):3689-3704, 20 May 1984.

[61] F. J. Wentz and D. K. Smith. A model function for the ocean-normalized radar cross section at $14 \mathrm{GHz}$ derived from NSCAT observations. J. Geophys. Res., 104(C5):1149911514, 15 May 1999. 


\section{TABLE LEGENDS}

Table 1: Summary of "obs" functions and background constraints used in the variational analysis. Subscript a refers to analyzed values (e.g., $V_{a}$ is the analyzed wind vector), subscript $b$ to background values, subscript o to observed values, and subscript $k$ to the kth ambiguity retrieved from scatterometer data. Other subscripts are defined in the table, and $\chi, \psi$ and $\zeta$ are velocity potential, streamfunction and relative vorticity, respectively. For clarity scaling factors are not included in this table. Complete definitions and our implementation are detailed in the text.

Table 2: Summary of the $\lambda$ weights and results for the sensitivity experiments described in the text. In all experiments listed $\lambda_{v w M}=1$. In addition to the values of the other $\lambda$ weights the table lists the total number of function evaluations $\left(N_{f}\right)$, the rms gradient of the objective function $\left(\nabla J\left(\times 10^{6}\right)\right)$, and the objective function itself $(J)$. The analysis speed $(|V|)$ and direction $(\theta)$ are given at the "obs" location.

Table 3: $\quad$ Statistics comparing surface wind analyses from optimal interpolation (OI) and 2dVAR with the background and the first analysis in the table. The OI analysis uses twice the nominal wind component error standard deviation $\left(s_{u}\right)$. In the remaining analyses, the $2 d-V A R \lambda$ weights are held fixed, excepting $\lambda_{C O N V}$ which is varied systematically. The statistics calculated are the mean and the rms of the wind speed for the field, the rms of the magnitude of the vector differences between the analyses and background (denoted increment), and the rms of the magnitude of the vector differences between the analyses and OI analysis (denoted difference).

Table 4: Differences between JPL and 2d-VAR ambiguity selection as a function of wind speed for 15 October-5 November 1996. The column labeled $N$ gives the number of comparisons in each wind speed bin. 


\section{FIGURE LEGENDS}

Fig. 1: Analyzed wind field for $1 / 2^{\circ}$ resolution, fully converged for the nominal case described in the text. Here, and in the figures which follow, the wind barbs are in knots and all results are presented on a $1^{\circ}$ grid. Also the color scale shown here (in $\mathrm{ms}^{-1}$ ) is used in the figures which follow.

Fig. 2: Statistical interpolation results using NOGAPS statistics.

Fig. 3: The effect of resolution. Results for 2, 1, 1/2 and $1 / 4^{\circ}$ resolution. The panel labeled "1/2 degree resolution" repeats the results shown in Fig. 1.

Fig. 4: Differences, scaled by 10 , from the result for $1 / 8^{\circ}$ resolution, for the results for 2,1 , $1 / 2$ and $1 / 4^{\circ}$ resolution (shown in Fig. 3). (In the first panel, and in future figures, results greater than $20 \mathrm{~ms}^{-1}$ are colored (yellow) as if they were $20 \mathrm{~ms}^{-1}$.)

Fig. 5: The sequence of results for increasing number of iterations for $1 / 2^{\circ}$ resolution, starting from a zero initial estimate.

Fig. 6: Differences (times 10) between the result using only 25 iterations at each resolution and the "fully" converged solutions (shown in Fig. 3) for $2,1,1 / 2$ and $1 / 4^{\circ}$ resolution.

Fig. 7: Solutions for different $\lambda$ weights. The nominal case is shown in the lower left panel. The $\lambda_{L A P}, \lambda_{D V}, \lambda_{V O R}$, and $\lambda_{D Y N}$ increase by a factor of 4 in the right column, while $\lambda_{\text {Conv }}$ increases by a factor of 4 in the upper row.

Fig. 8: Solutions for setting some of the $\lambda$ weights to zero. The nominal case is shown in the lower left panel. The $\lambda_{\text {DYN }}$ is set to zero in the right column, while $\lambda_{\text {DIV }}$ and $\lambda_{\text {VOR }}$ are set to zero in the upper row. 
Fig. 9: Differences (times 10) from the solution for $1 / 4^{\circ}$ resolution for runs at $1^{\circ}$ and $1 / 2^{\circ}$ using bilinear and bicubic interpolation procedures.

Fig. 10: Effect of adding a second surface wind observation. The difference (times 10) of the solution for two observations minus the sum of the individual solutions is shown in the lower right panel.

Fig. 11: JPL-selected ambiguities from rev 2607.

Fig. 12: Background wind field, time interpolated to 1930 UTC February 15, 1997 from sixhourly ECMWF $10 \mathrm{~m}$ wind fields. Wind speed is contoured every $2 \mathrm{~ms}^{-1}$ and NSCAT "obs" locations are plotted as small black dots.

Fig. 13: Wind field analysis from TAP with the standard deviation of "obs" error inflated by a factor of two. Wind speed is contoured every $2 \mathrm{~ms}^{-1}$ and NSCAT "obs" locations are plotted as small black dots.

Fig. 14: Wind field analysis increments from TAP corresponding to Fig. 13. Wind speed is contoured every $2 \mathrm{~ms}^{-1}$ and NSCAT "obs" locations are plotted as small black dots.

Fig. 15: 2d-VAR observation operators evaluated for one WVC using $\sigma^{0}$ values directly (upper panel), and ambiguous winds (middle panel). For reference, the corresponding twowind observation operator in use at ECMWF is shown (lower panel) (Stoffelen and Anderson (1997 [54])).

Fig. 16: The rms fit of analyses to NSCAT wind observations as a function of $\log _{10}(\lambda)$ weight for the October 27, 1996 case (rev 1025). Curves for analyses using ambiguous wind (w) and $\sigma^{0}(s)$ observations are shown. 
Fig. 17: NCEP background for winds and $\sigma^{0}$ analyses, valid at 2230 UTC October 27, 1996.

Fig. 18: Comparison of VAM analyses from wind and $\sigma^{0}$ observations for $\lambda_{A M B}=4$ and $\lambda_{N R C S}=1 / 4$, valid at 2230 UTC October 27,1996 . The upper panels are analyses and lower panels are analysis increments (analysis-background times ten).

Fig. 19: North Atlantic overview at 1315 UTC 26 Oct 1996 including GOES-8 IR brightness temperature (K). NSCAT WVC locations are marked by fine black dots. Heavy blue dots mark WVC's where JPL and 2d-VAR ambiguity selection differ.

Fig. 20: ECMWF (thin red barbs) and 2d-VAR (thick black barbs) analyses in region $A$ with GOES-8 IR brightness temperature. See Fig. 19 for the location of region A. NSCAT WVC locations are marked by fine black dots. Blue circles with white centers mark WVCS where JPL and 2d-VAR ambiguity selection differ.

Fig. 21: All JPL ambiguities and GOES-8 IR brightness temperature in subregion A1. See Fig. 20 for location of subregion A1. Blue circles with white centers mark WVCs where JPL and 2d-VAR ambiguity selection differ.

Fig. 22: JPL (a) and 2d-VAR (b) selected ambiguities, and GOES-8 IR brightness temperature in subregion A1. See Fig. 20 for location of subregion A1. Blue circles with white centers mark WVCs where JPL and 2d-VAR ambiguity selection differ. 


\section{TABLES}

Table 1:

\begin{tabular}{lcl}
\hline \hline Term & Expression & \multicolumn{1}{c}{ Description } \\
\hline$J_{\mathrm{CONV}}$ & $\sum\left(\boldsymbol{V}_{a}-\boldsymbol{V}_{o}\right)^{2}$ & for the conventional data \\
$J_{\mathrm{AMB}}$ & $\sum \prod_{k}\left\{1-\exp \left[-\left(\boldsymbol{V}_{a}-\boldsymbol{V}_{k}\right)^{2}\right]\right\}$ & for the ambiguous winds \\
$J_{\mathrm{SPD}}$ & $\sum\left[\left|\boldsymbol{V}_{a}\right|-\operatorname{rms}\left(\left|\boldsymbol{V}_{o}\right|\right)\right]^{2}$ & for the scatterometer wind speed \\
$J_{\mathrm{NRCS}}$ & $\sum\left(\sigma^{0}{ }_{a}-\sigma^{0}{ }_{o}\right)^{2}$ & for the backscatter \\
\hline & $\int\left(\boldsymbol{V}_{a}-\boldsymbol{V}_{b}\right)^{2}$ & Background constraints \\
\hline$J_{\mathrm{VWM}}$ & $\int\left[\nabla^{2}\left(\chi_{a}-\chi_{b}\right)\right]^{2}$ & on the vector wind magnitude \\
$J_{\mathrm{LAP}}$ & $\left.\left.\int u_{a}-u_{b}\right)\right]^{2}+\int\left[\nabla^{2}\left(v_{a}-v_{b}\right)\right]^{2}$ & on the Laplacian of the wind components \\
$J_{\mathrm{DIV}}$ & $\int\left[\nabla^{2}\left(\psi_{a}-\psi_{b}\right)\right]^{2}$ & on the divergence \\
$J_{\mathrm{VOR}}$ & $\int\left(\partial \zeta_{a} / \partial t-\partial \zeta_{b} / \partial t\right)^{2}$ & on the vorticity tendency \\
$J_{\mathrm{DYN}}$ &
\end{tabular}

Table 2:

\begin{tabular}{lrrrrrrrrrr}
\hline \hline Run & $\lambda_{\text {CONY }}$ & $\lambda_{\text {LAP }}$ & $\lambda_{\text {DIV }}$ & $\lambda_{\text {VOR }}$ & $\lambda_{\text {DYN }}$ & $|V|$ & $\theta$ & $N_{f}$ & $\nabla J$ & $J$ \\
\hline Nominal & 80 & 4 & 16 & 4 & 64 & 18.65 & 210 & 994 & 127 & 12937 \\
"Obs" + & 320 & 4 & 16 & 4 & 64 & 25.73 & 210 & 1060 & 1822 & 18715 \\
Constraints + & 80 & 16 & 64 & 16 & 256 & 11.78 & 209 & 1060 & 378 & 21398 \\
Background - & 320 & 16 & 64 & 16 & 256 & 20.83 & 210 & 1060 & 39460 & 41044 \\
w/o DIV, VOR & 80 & 4 & 0 & 0 & 64 & 21.91 & 210 & 1060 & 2188 & 9278 \\
w/o DYN & 80 & 4 & 16 & 4 & 0 & 21.64 & 210 & 498 & 119 & 10036 \\
LAP only & 80 & 4 & 0 & 0 & 0 & 25.17 & 210 & 513 & 107 & 5793 \\
\hline
\end{tabular}


Table 3:

\begin{tabular}{lrrrr}
\hline \hline Name & mean & rms & increment & difference \\
\hline Background & 8.788 & 9.287 & - & - \\
OI analysis, $s_{u} \times 2$ & 9.508 & 10.187 & 2.716 & - \\
2d-VAR analysis, $\lambda_{C O N V}=5$ & 9.510 & 10.183 & 2.726 & 0.584 \\
2d-VAR analysis, $\lambda_{C O N V}=10$ & 9.601 & 10.277 & 2.942 & 0.739 \\
2d-VAR analysis, $\lambda_{C O N V}=20$ & 9.675 & 10.351 & 3.109 & 0.935 \\
\hline \hline
\end{tabular}

Table 4:

\begin{tabular}{crr}
\hline \hline Wind Speed $\left(m s^{-1}\right)$ & $N$ & $\%$ Different \\
\hline $0-2$ & 260521 & 18.379 \\
$2-4$ & 836017 & 11.169 \\
$4-16$ & 5867770 & 3.807 \\
$>16$ & 142815 & 1.438 \\
\hline All & 7107123 & 5.141 \\
\hline
\end{tabular}




\section{FIGURES}

Fig. 1:

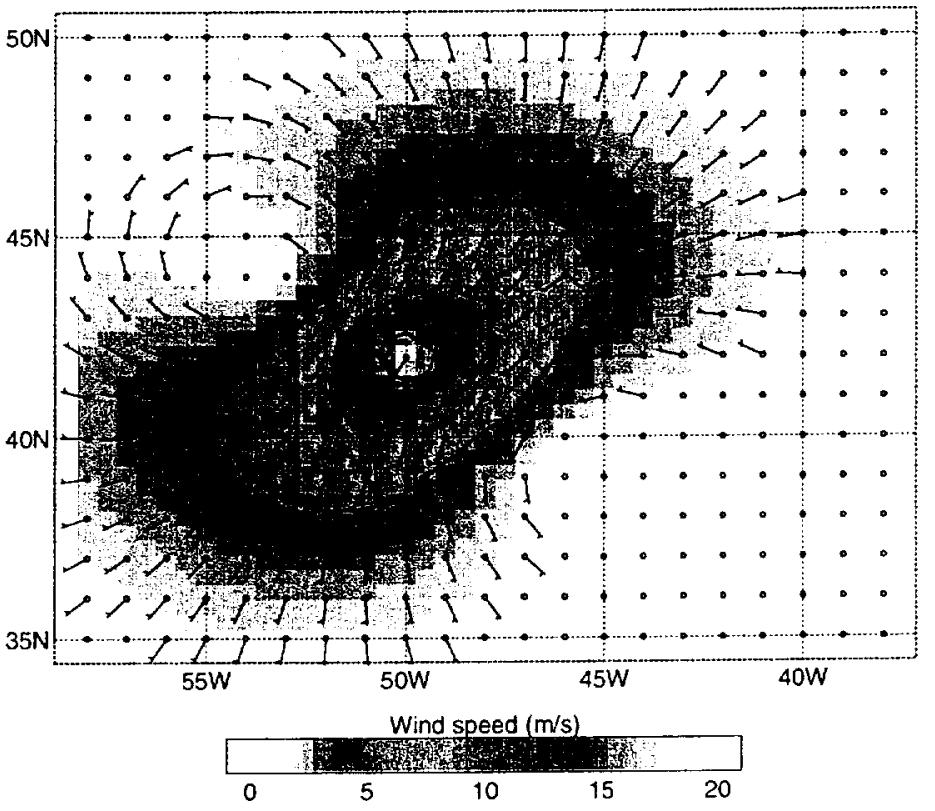

Fig. 2:

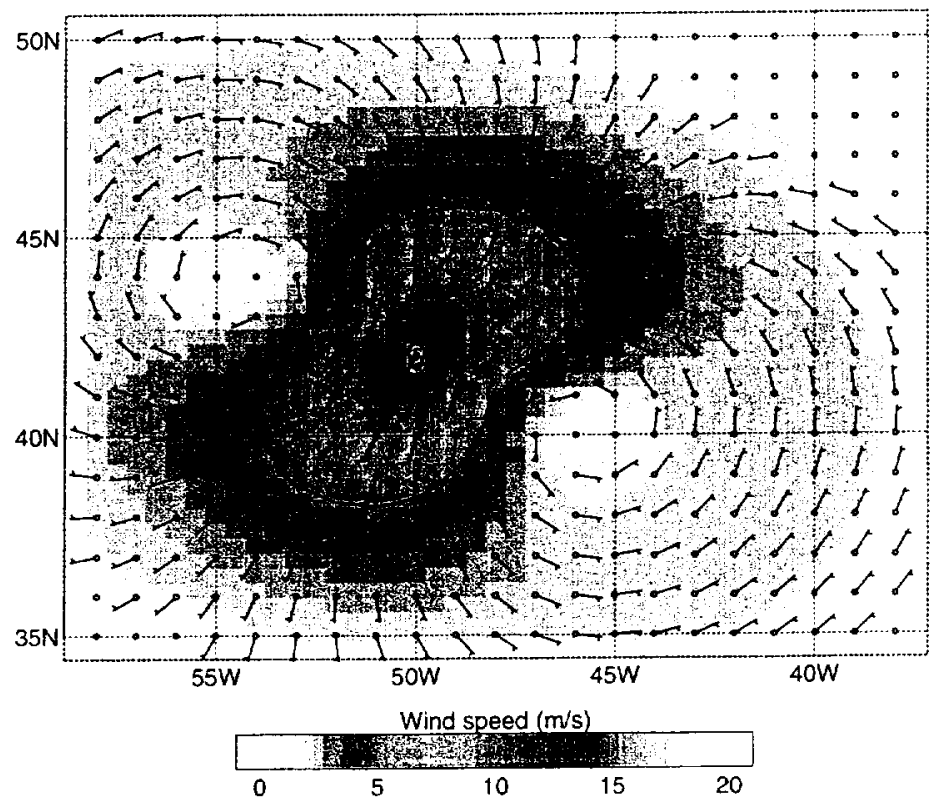


Fig. 3:
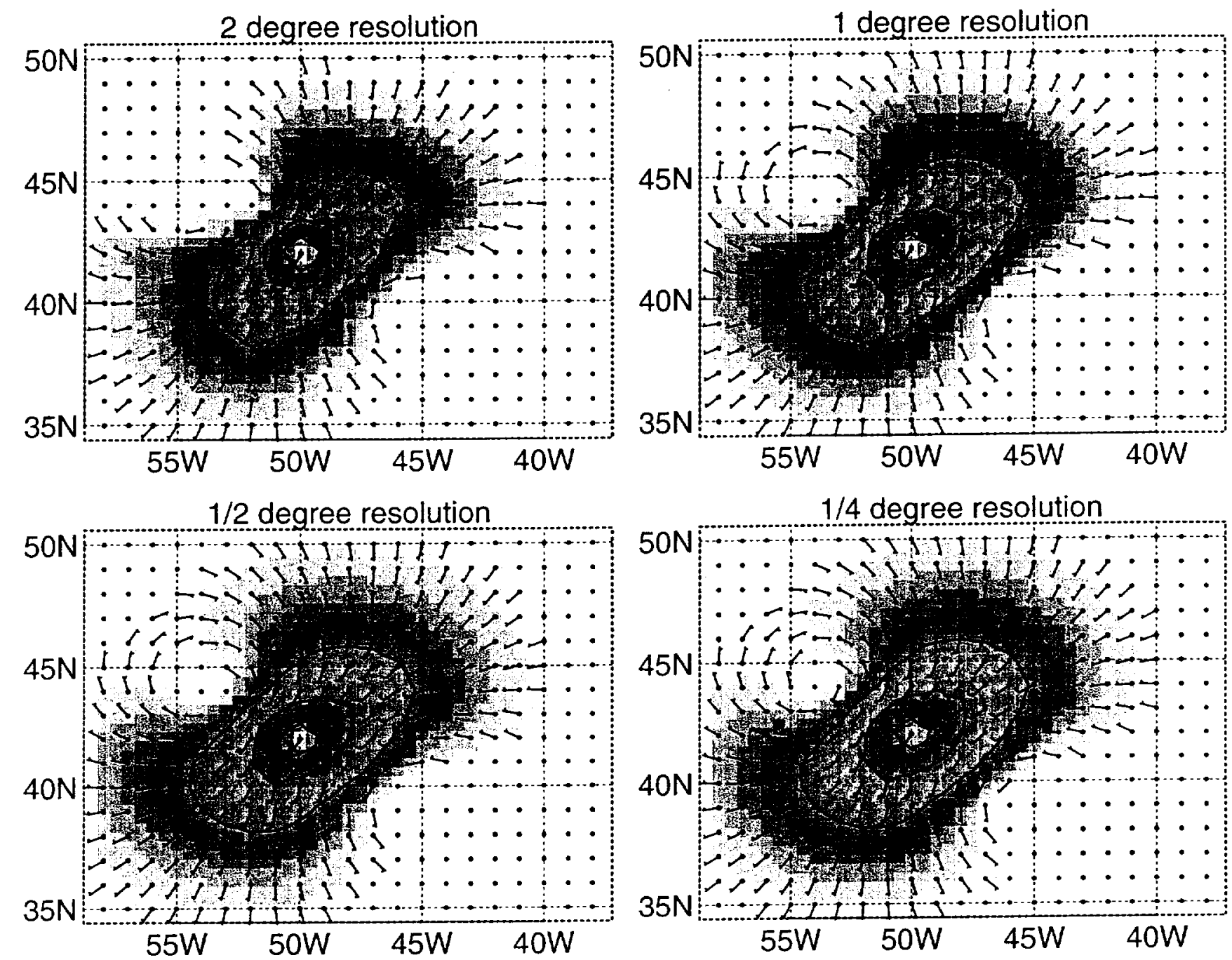
Fig. 4:
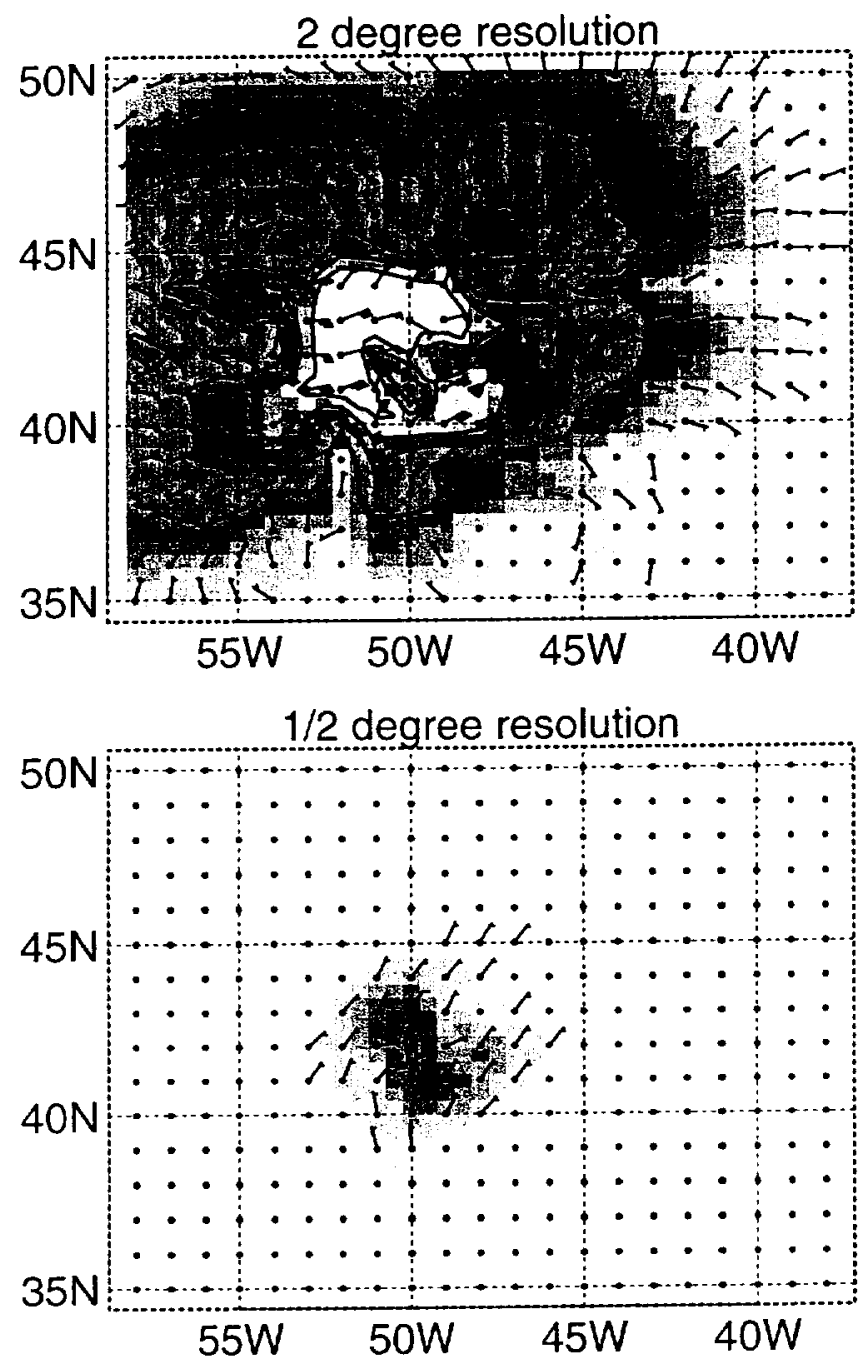

1 degree resolution

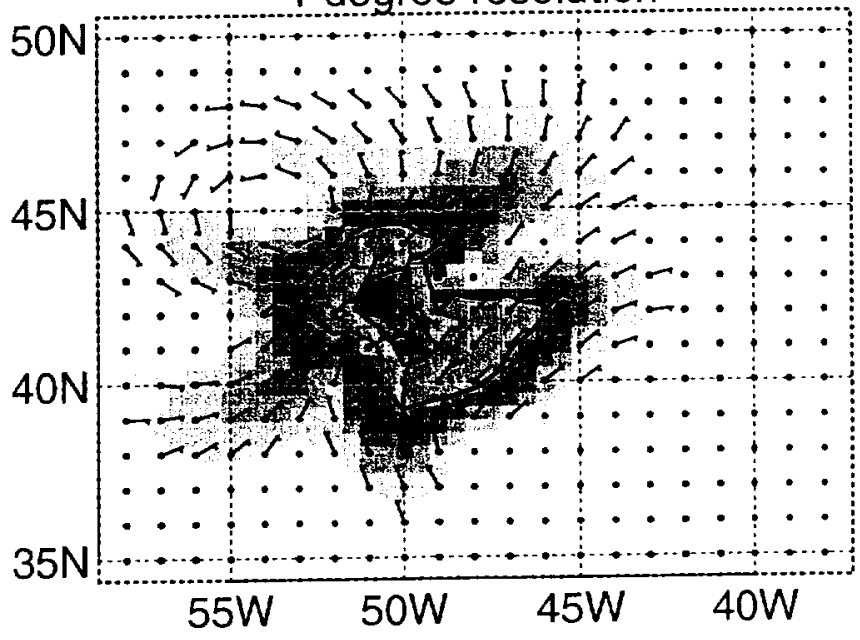

$1 / 4$ degree resolution

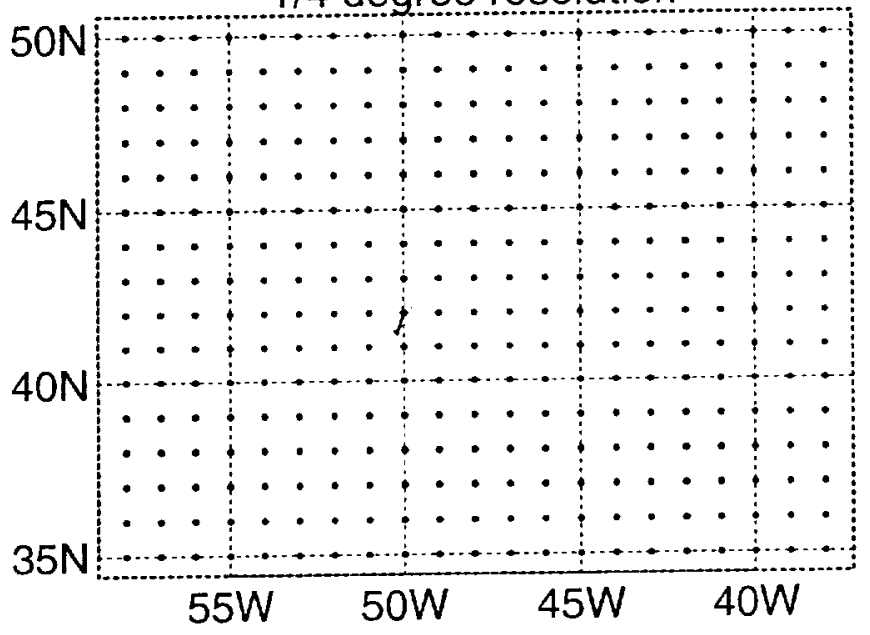


Fig. 5:
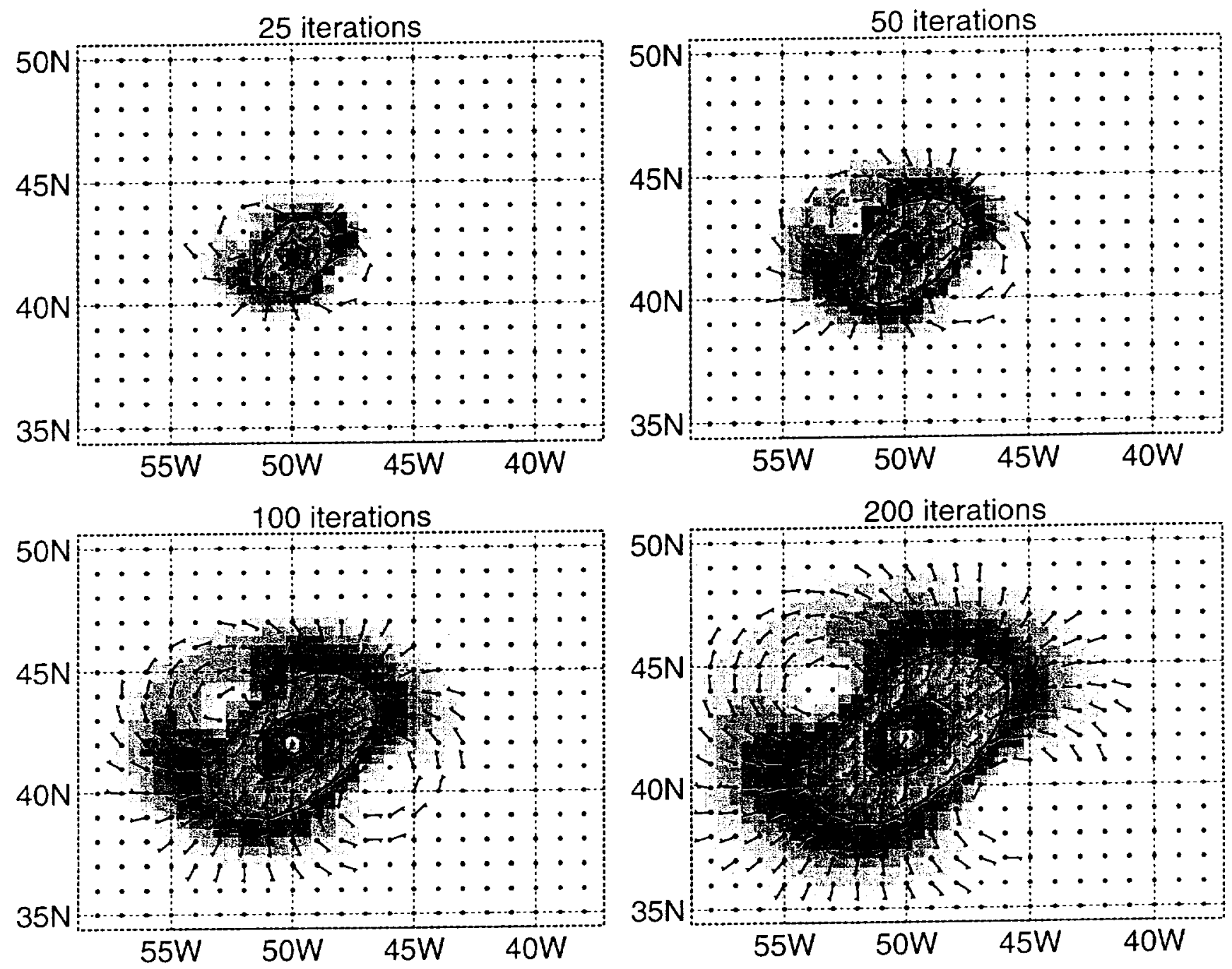
Fig. 6:

2 degree resolution

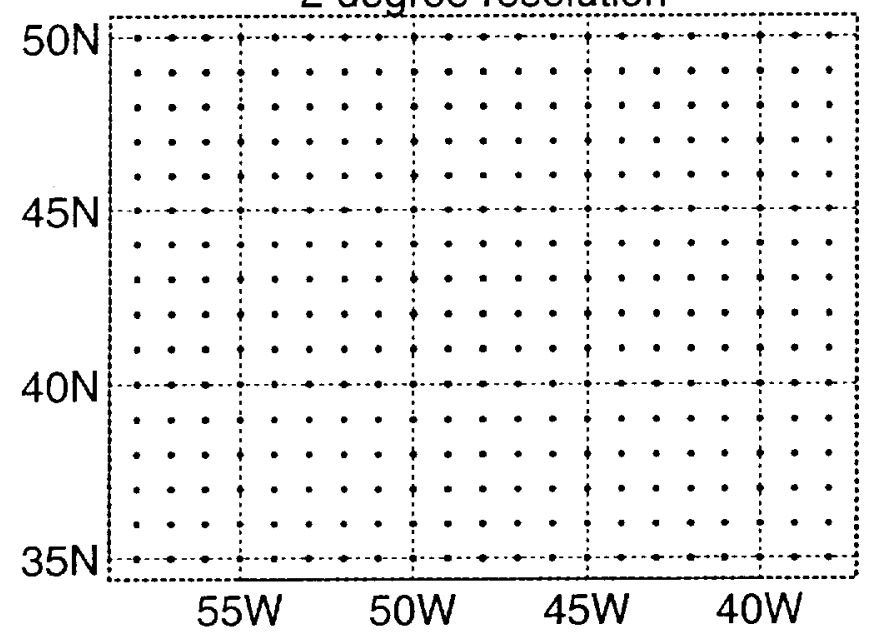

$1 / 2$ degree resolution

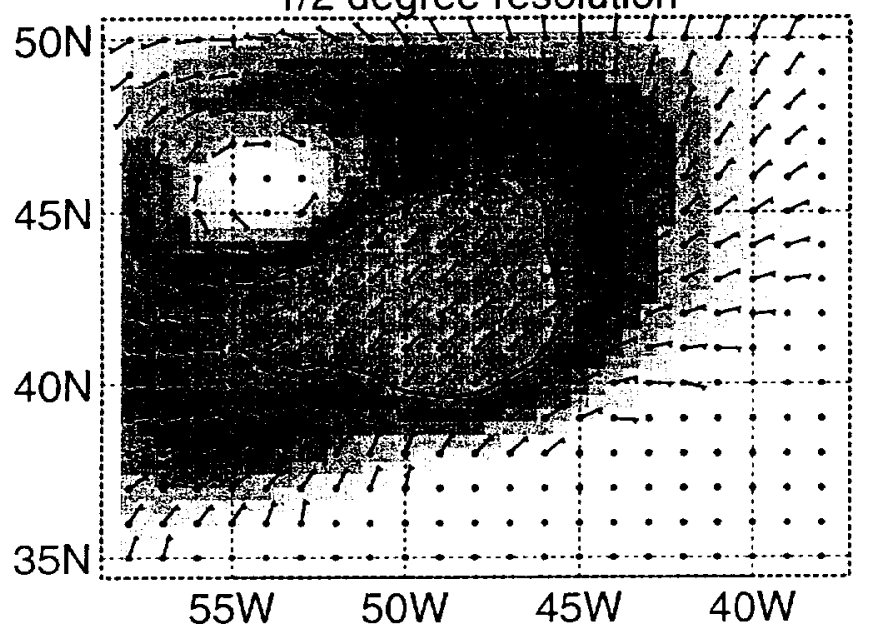

1 degree resolution

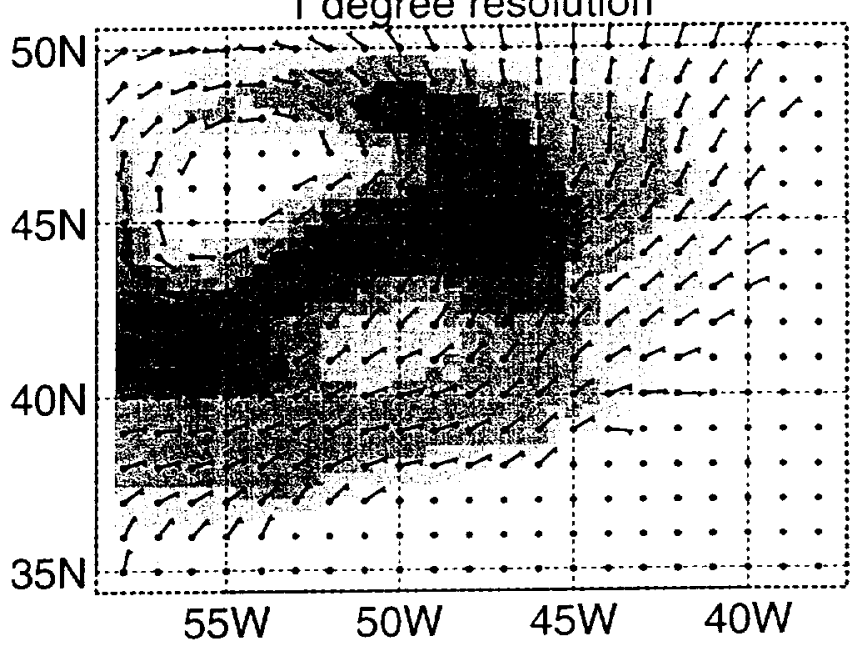

$1 / 4$ degree resolution

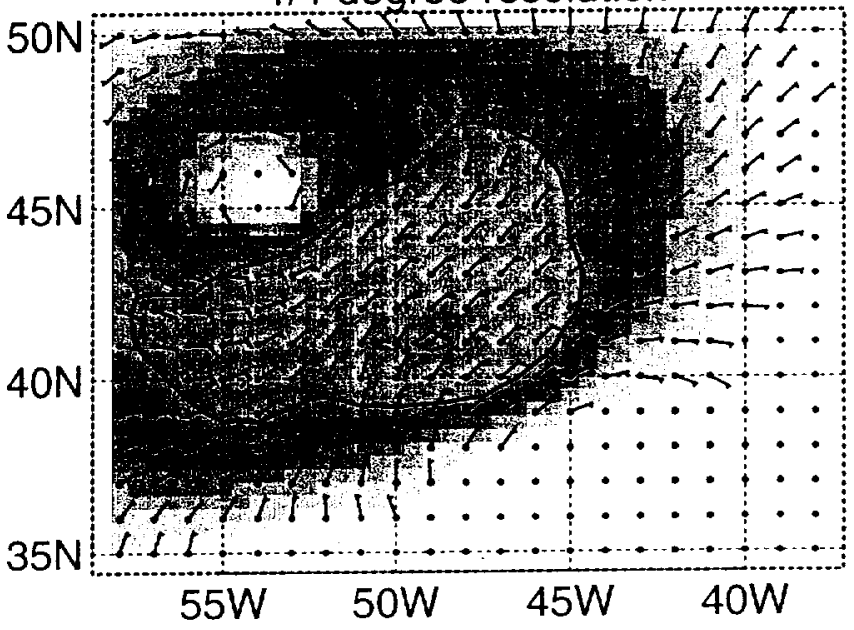


Fig. 7:

"Obs" +

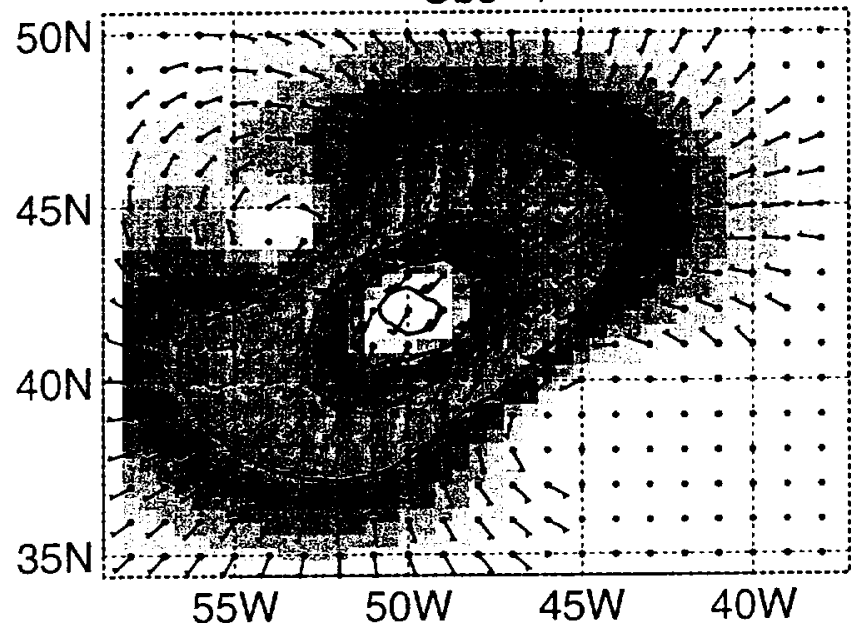

Nominal

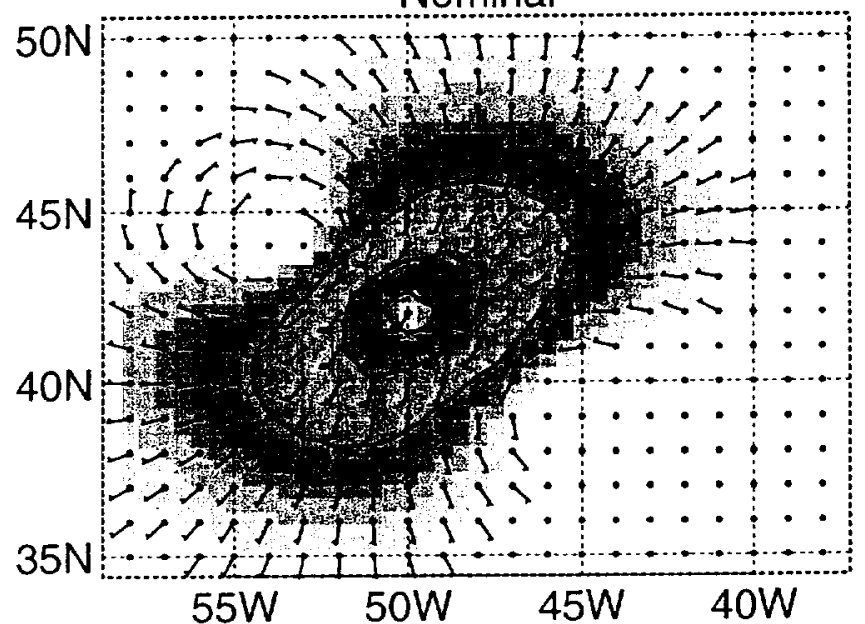

Background -

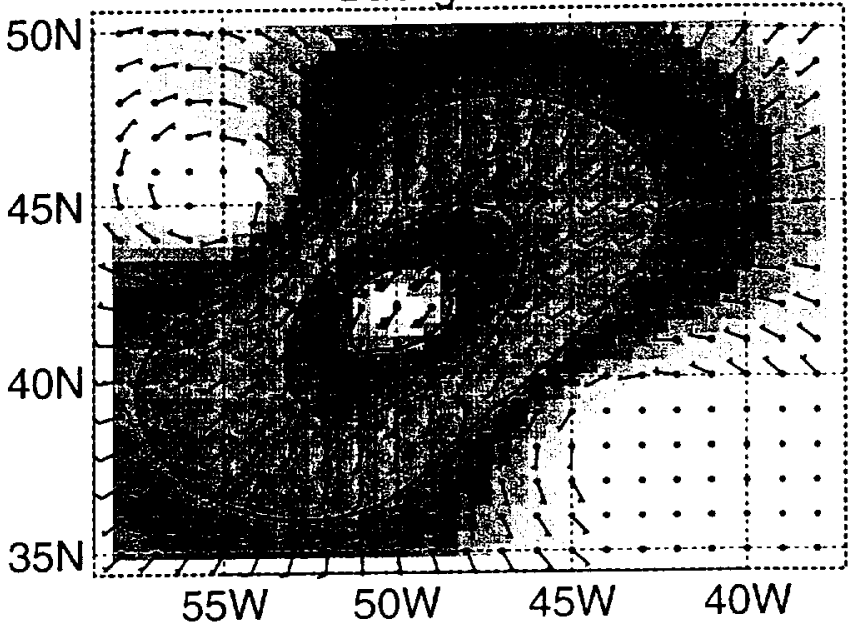

Constraints +

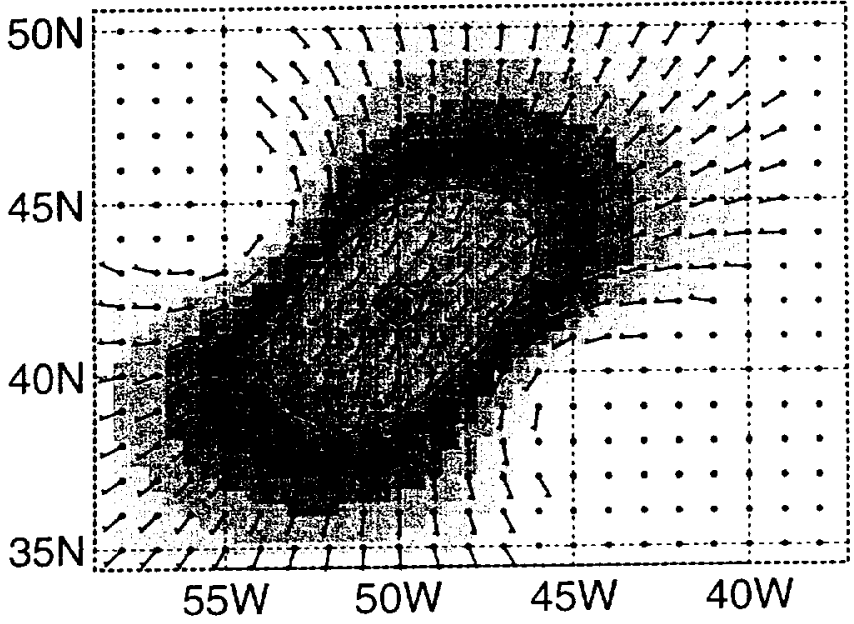


Fig. 8:
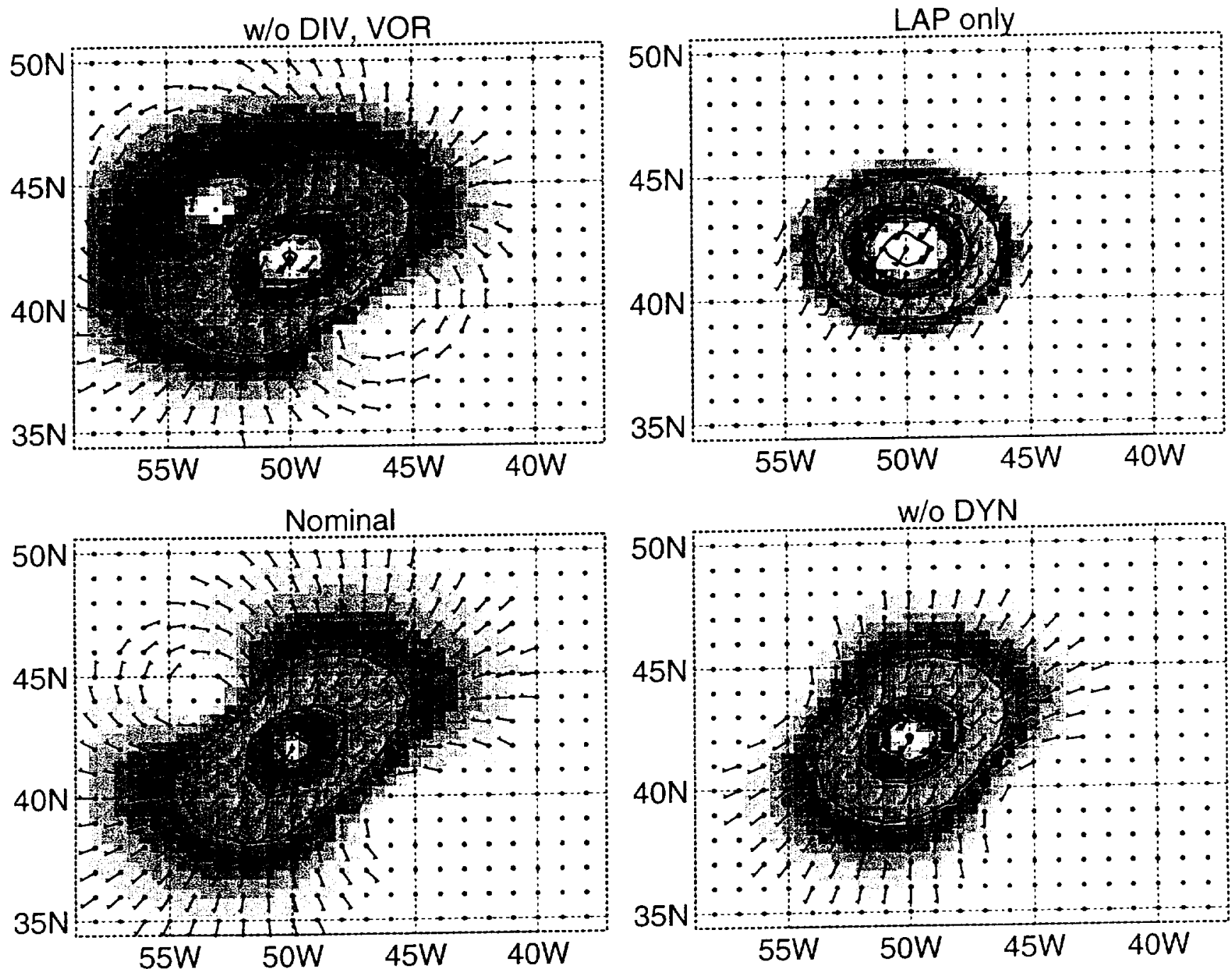
Fig. 9:
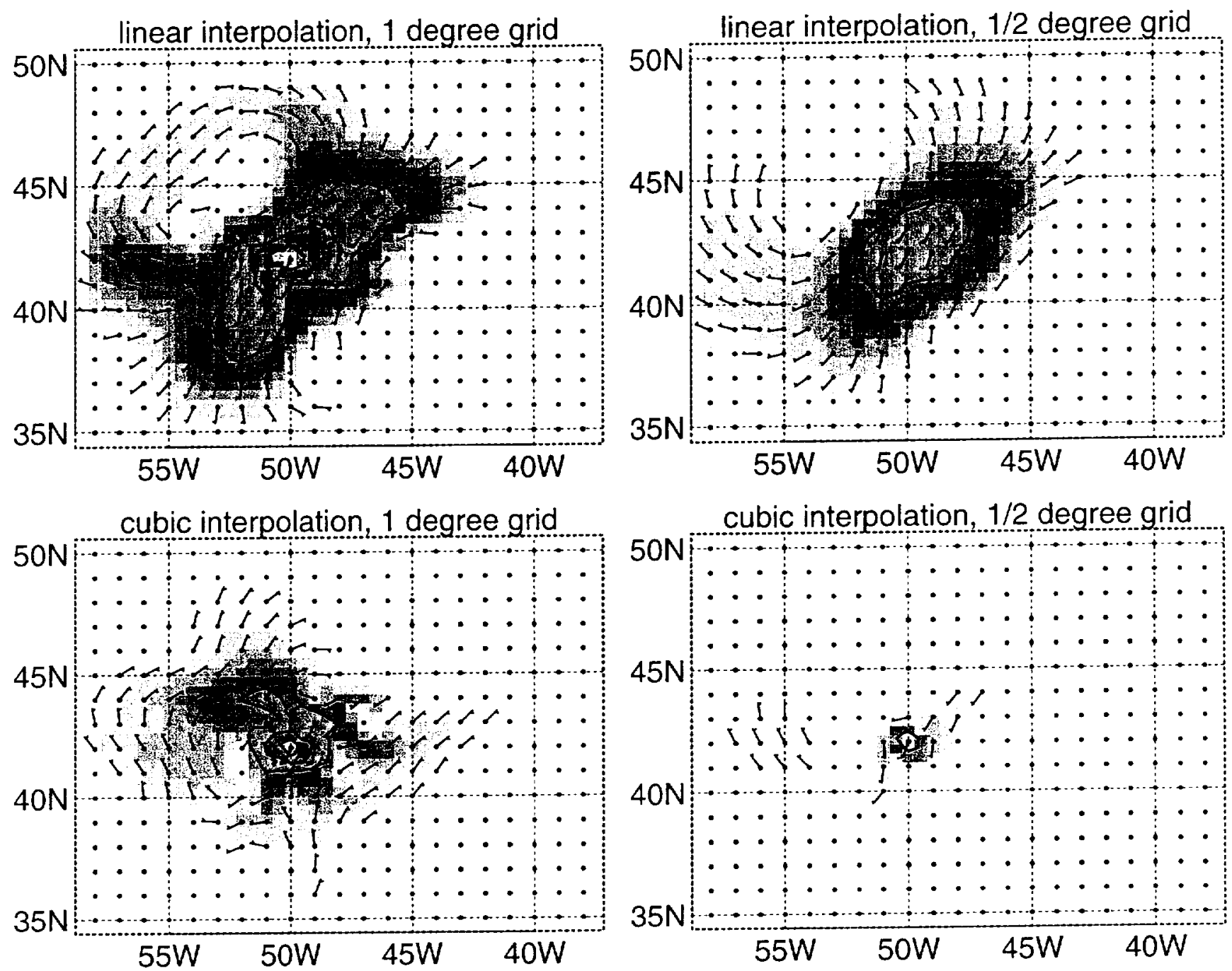
Fig. 10:

a second observation

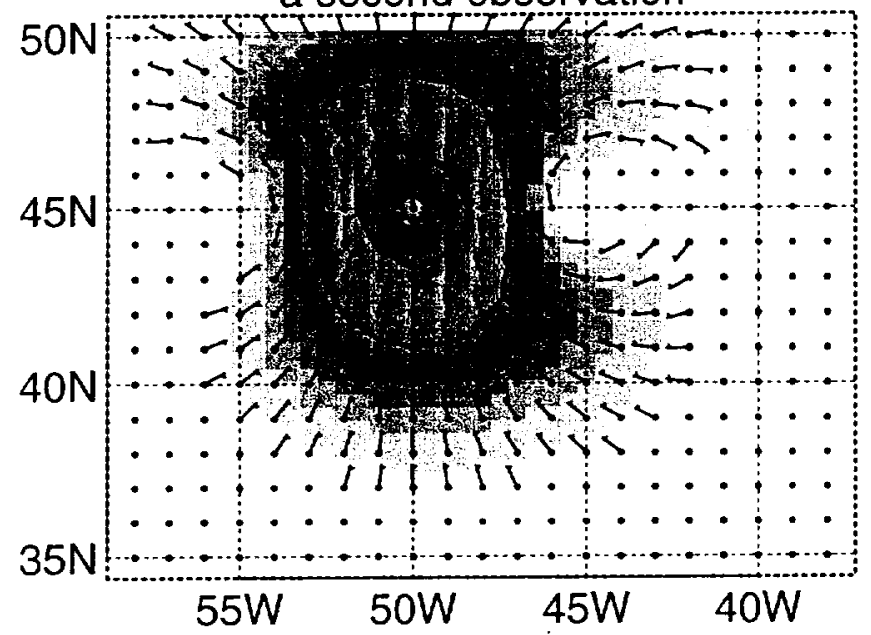

sum of solutions

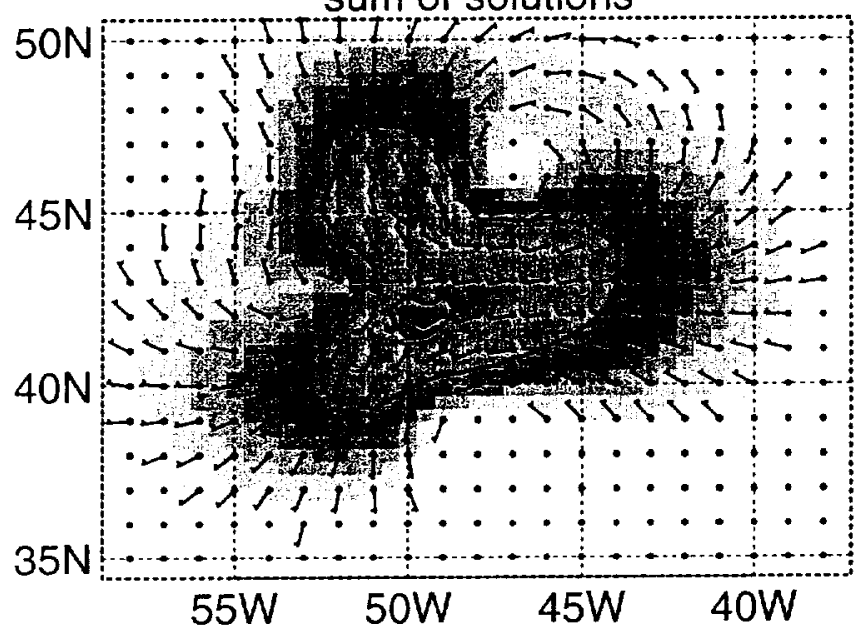

two observations

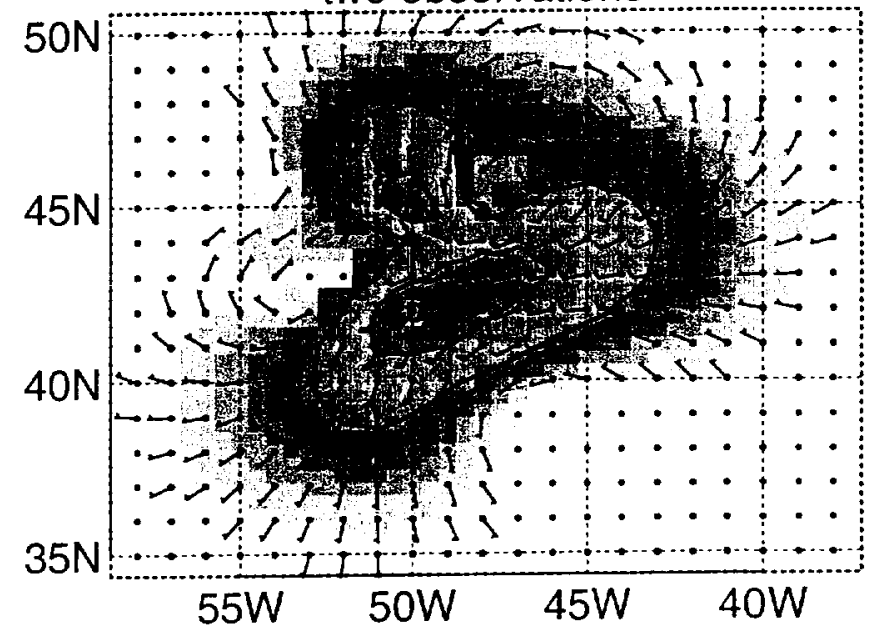

difference $\left({ }^{*} 10\right)$

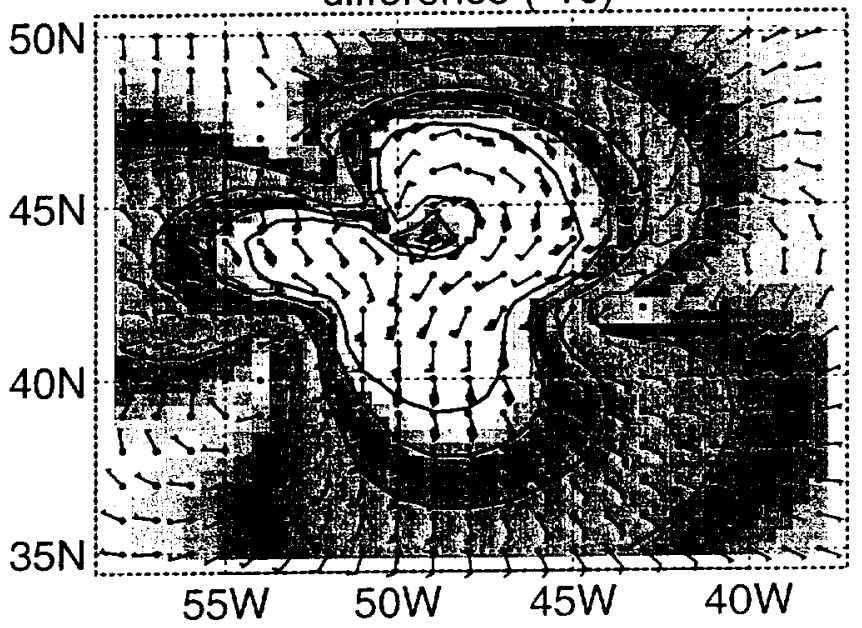


Fig. 11:

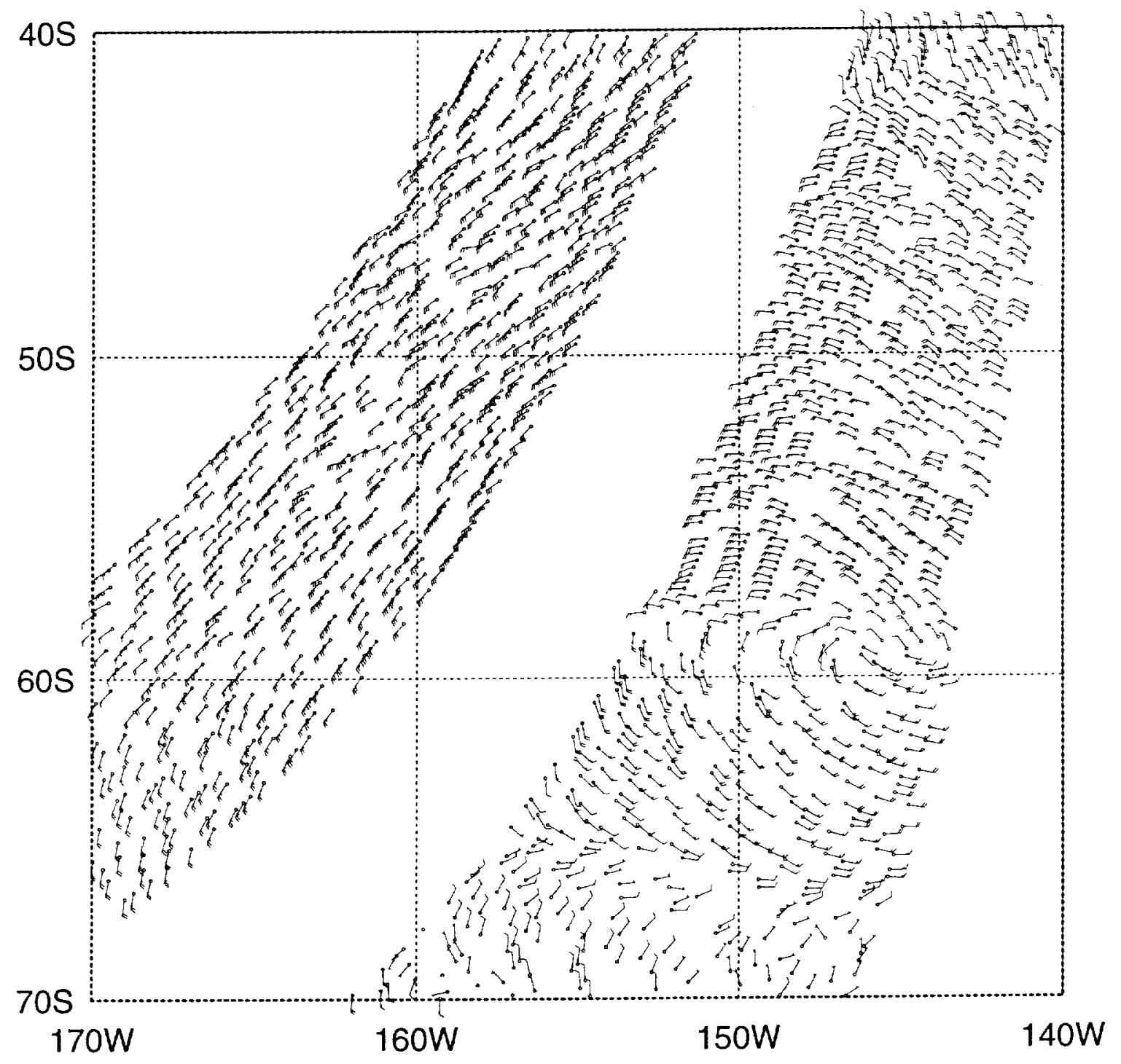


Fig. 12:

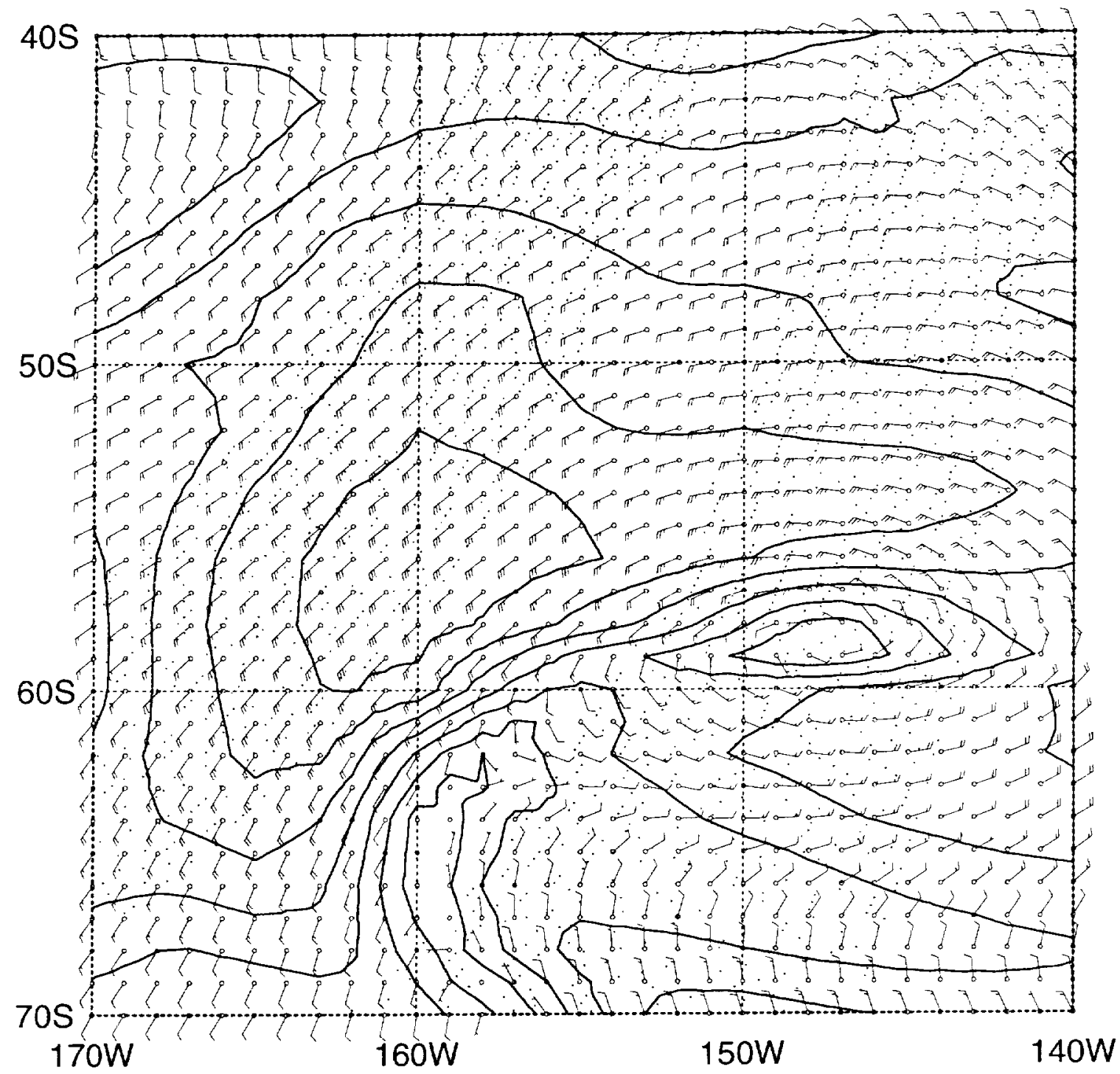


Fig. 13:

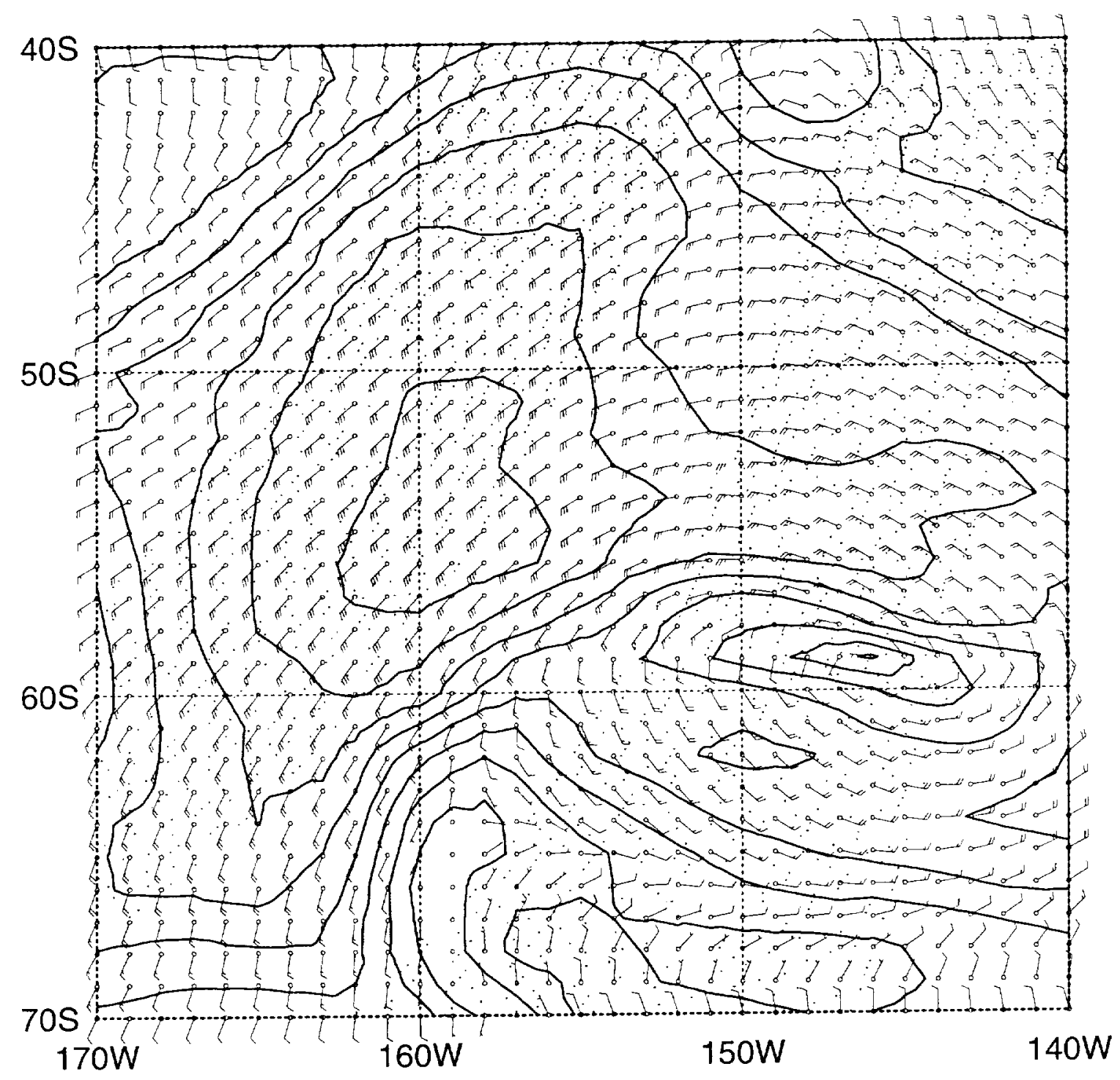


Fig. 14:

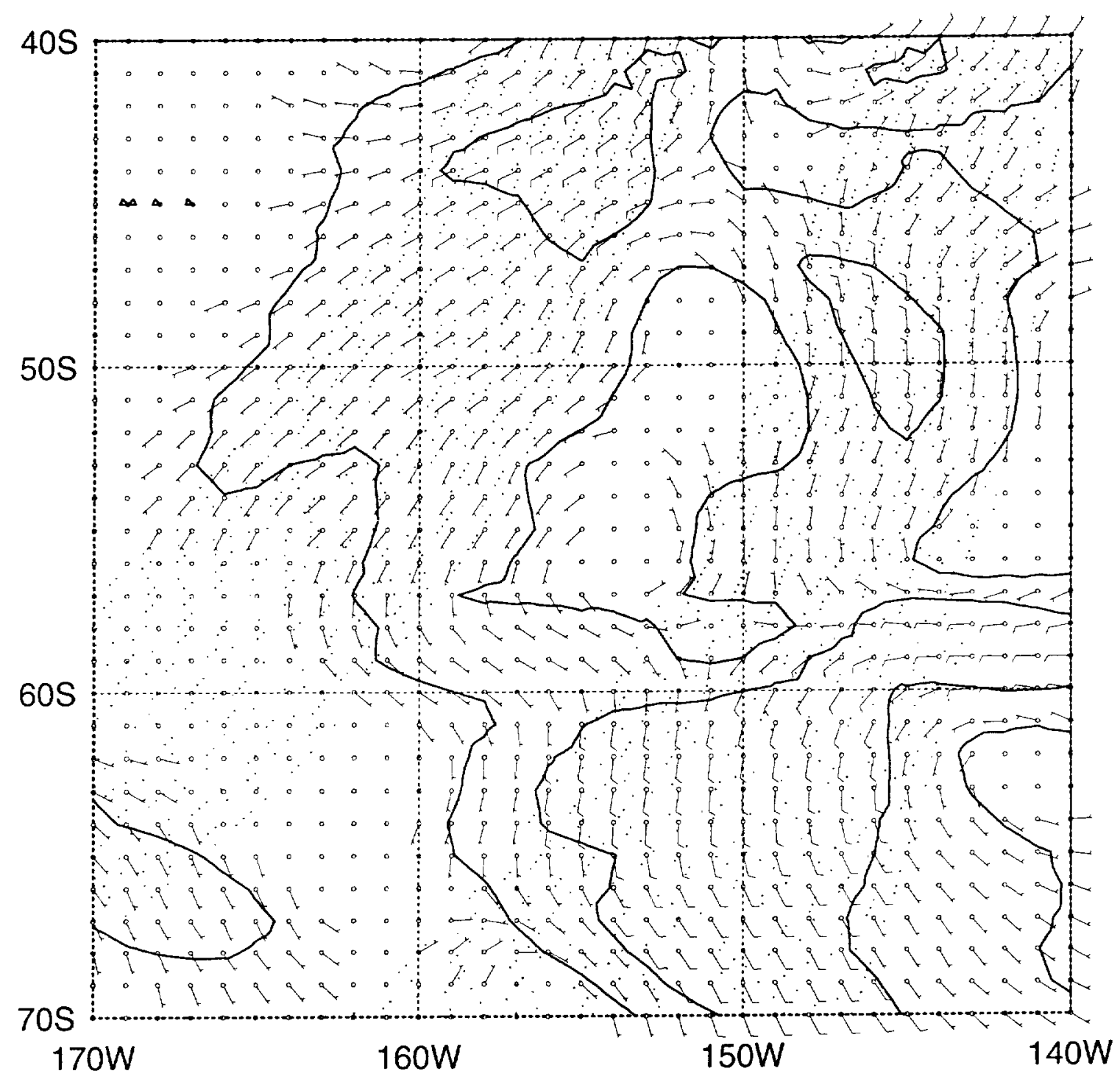


Fig. 15:
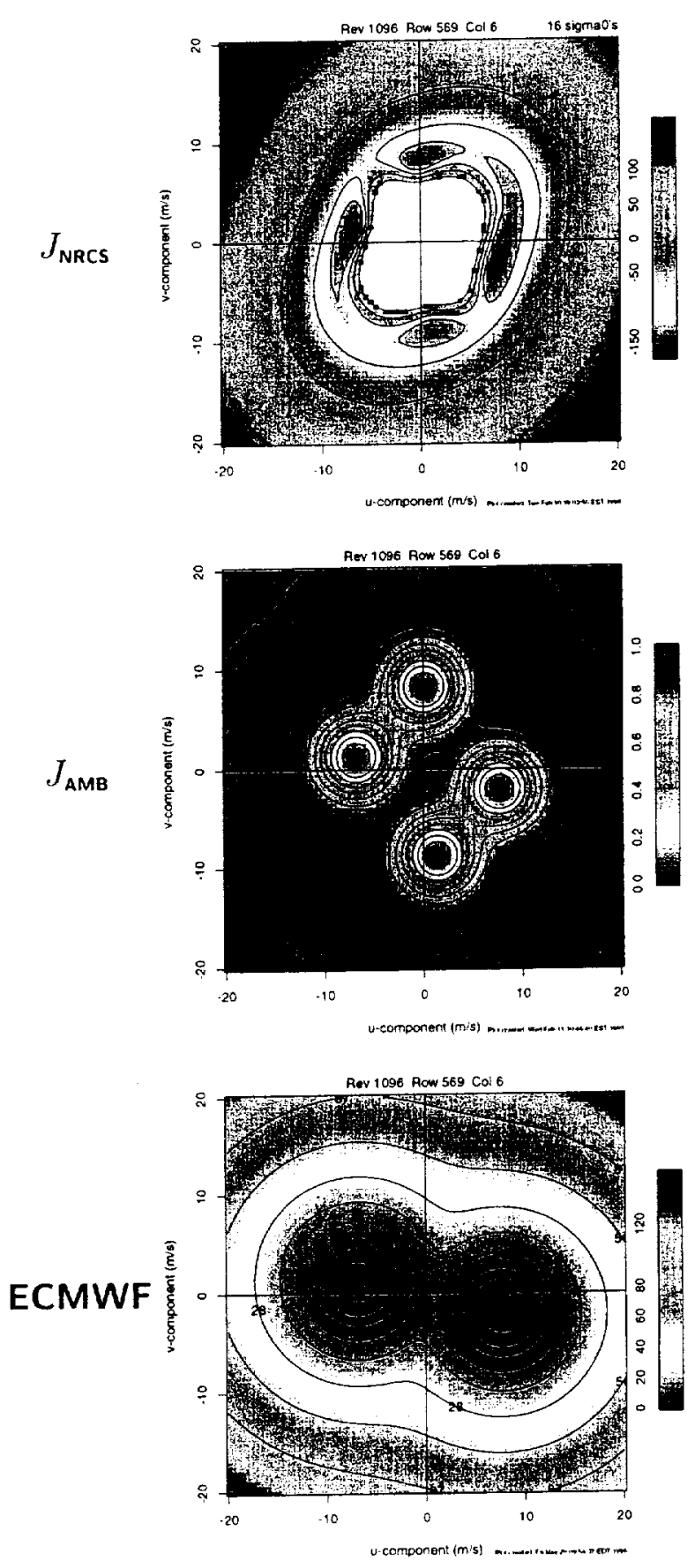
Fig. 16:

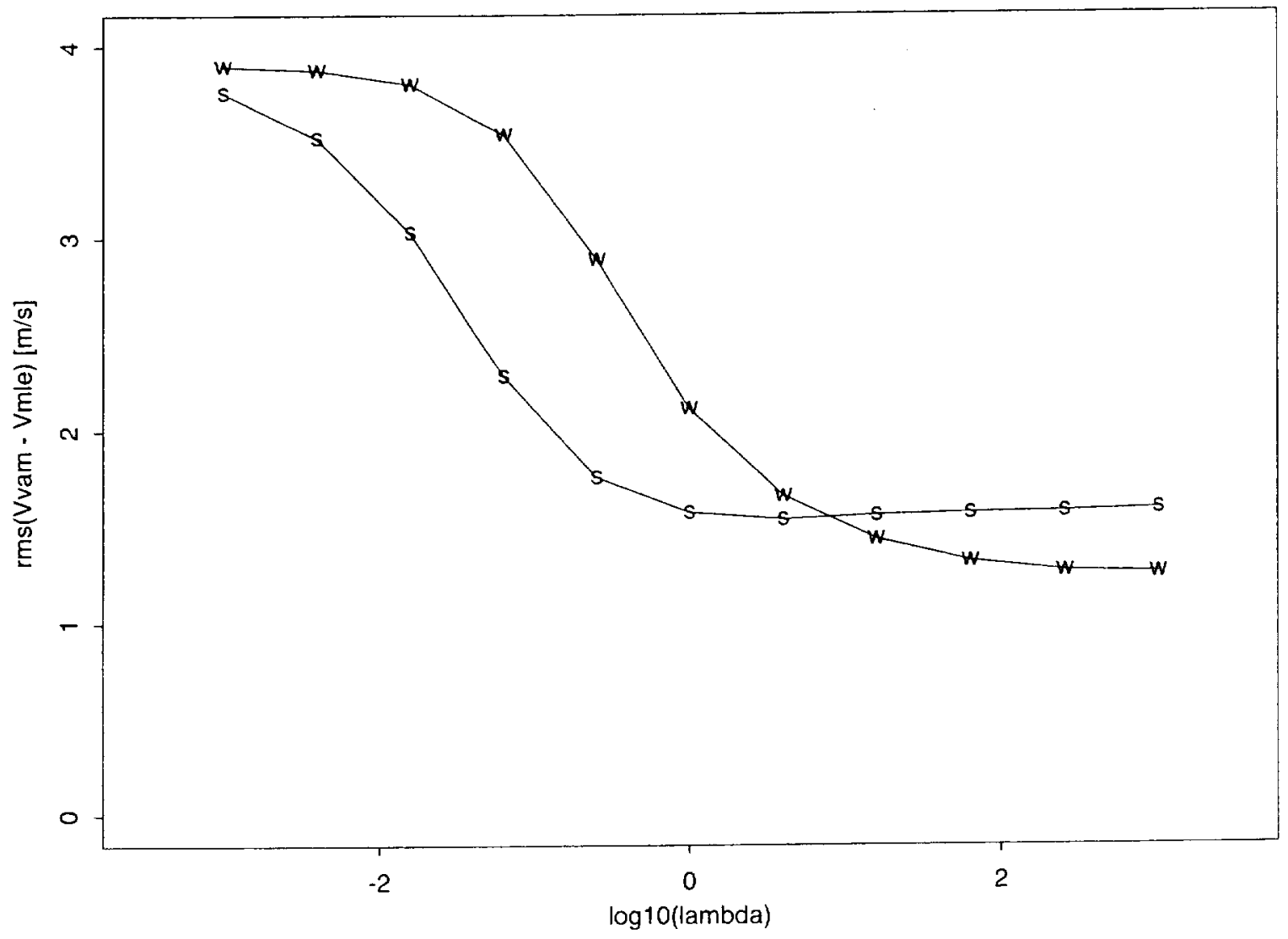


Fig. 17:

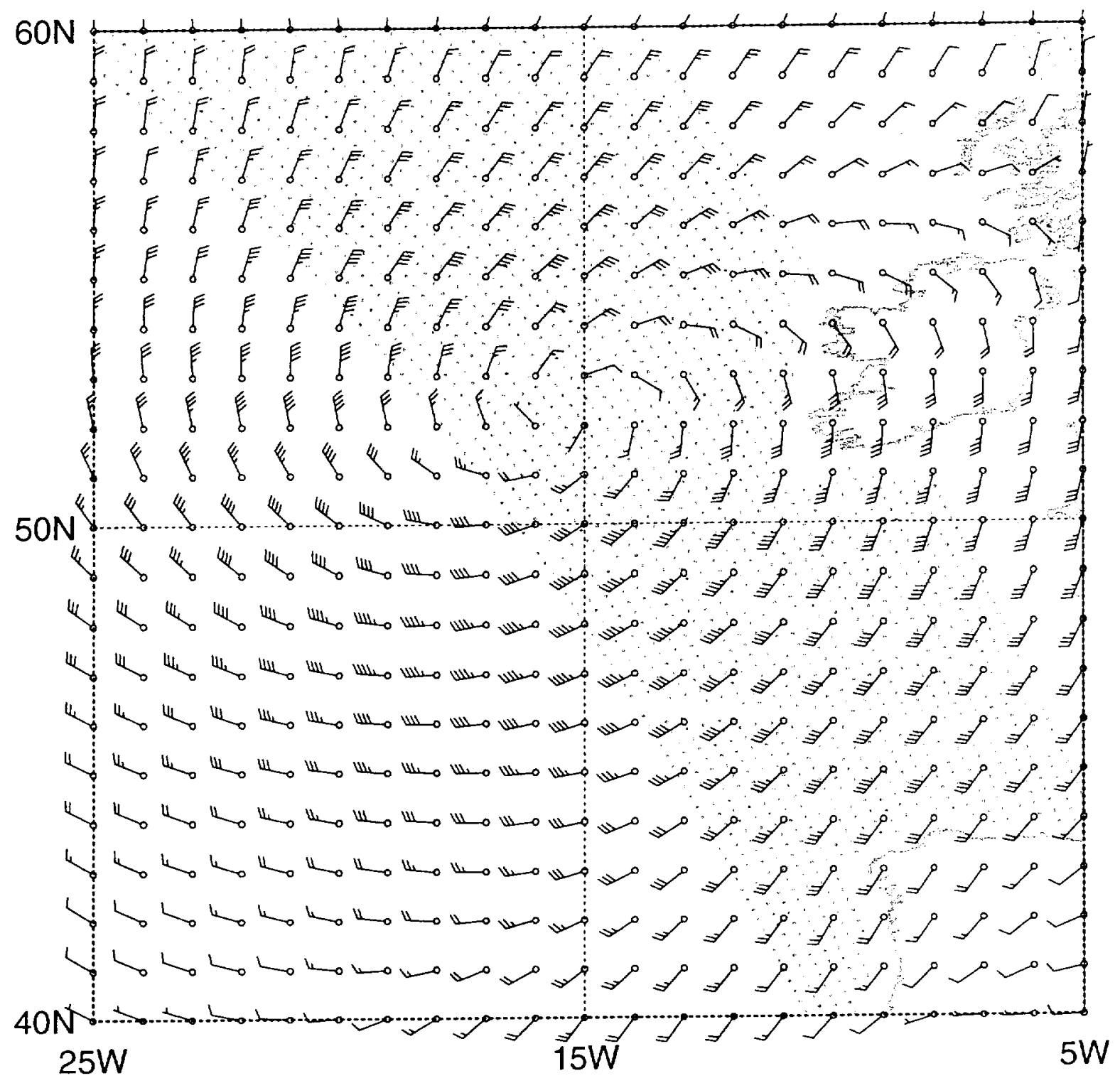


Fig. 18:

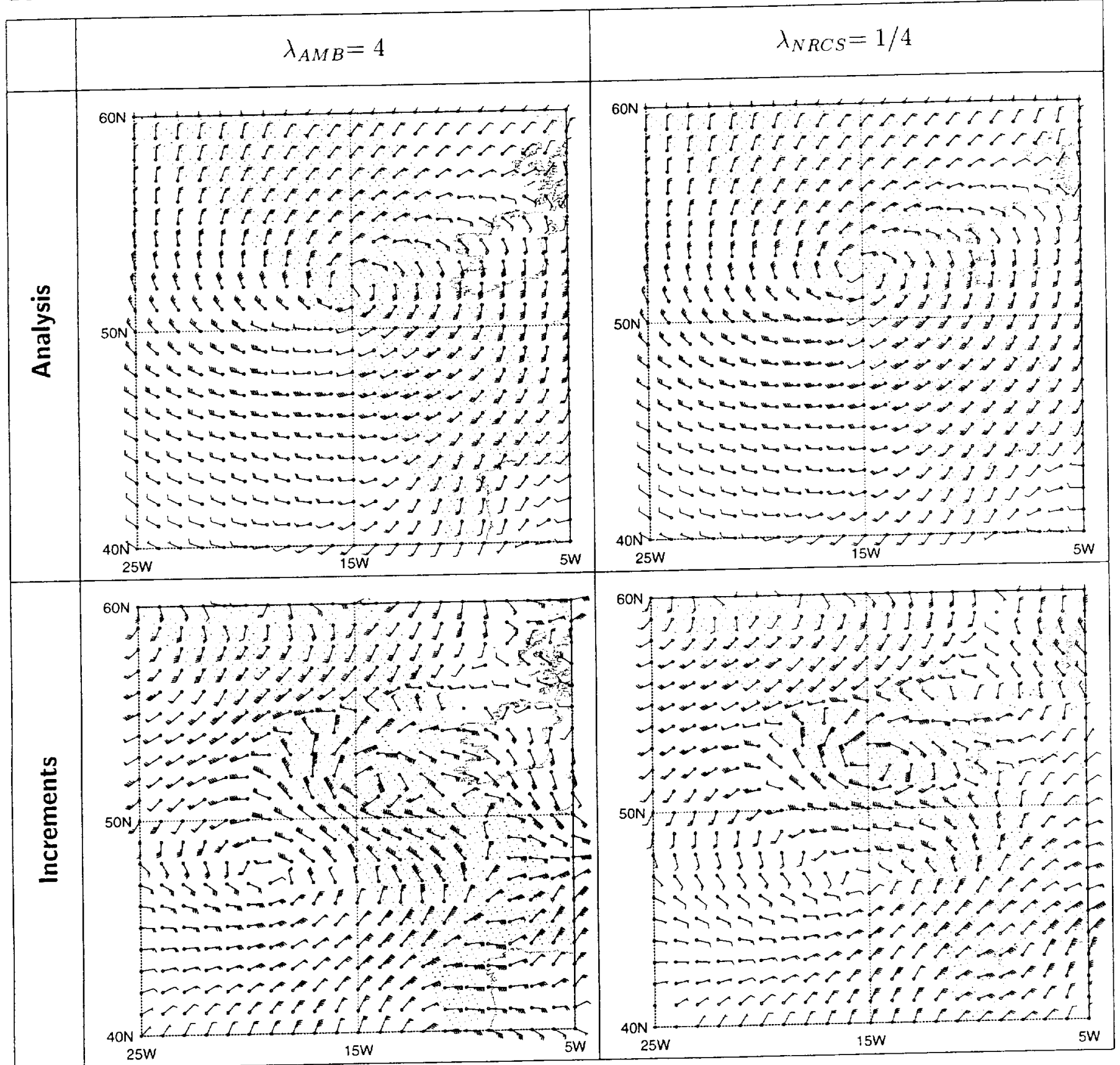


Fig. 19:

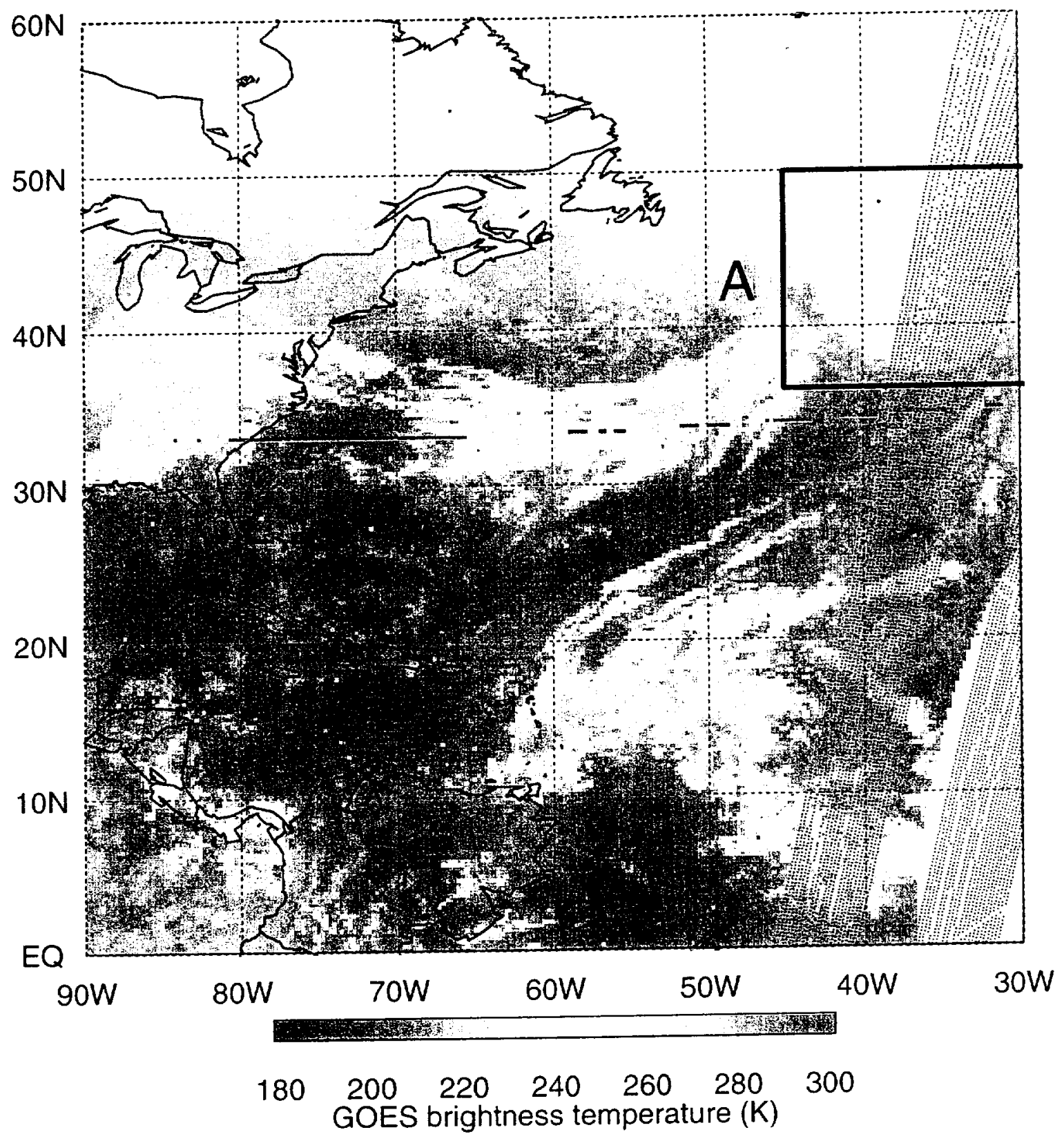


Fig. 20:

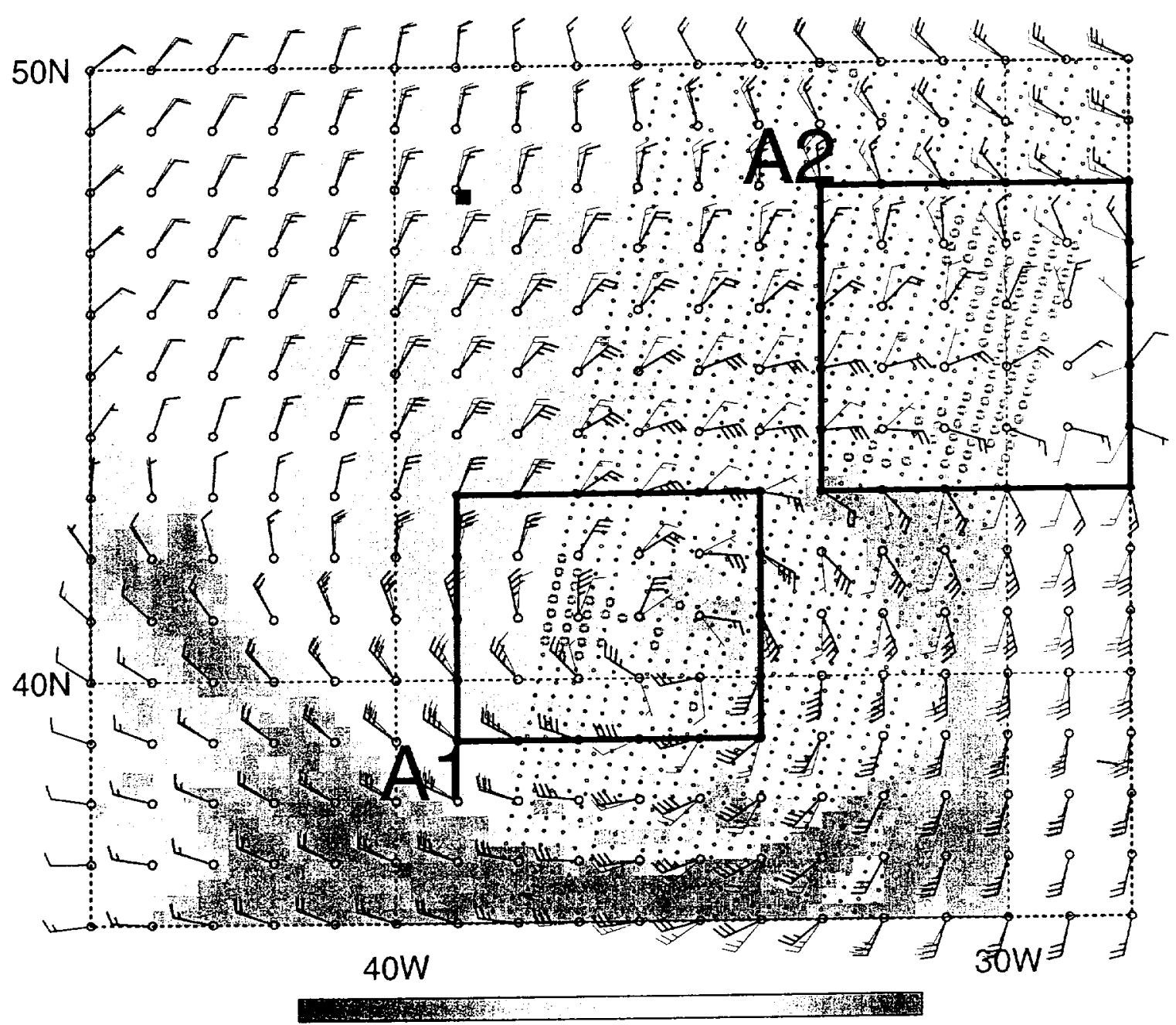

$180200 \begin{array}{cccc}220 & 240 & 260 & 280 \\ \text { GOES brightness temperature }(\mathrm{K}) & 300\end{array}$ 
Fig. 21:

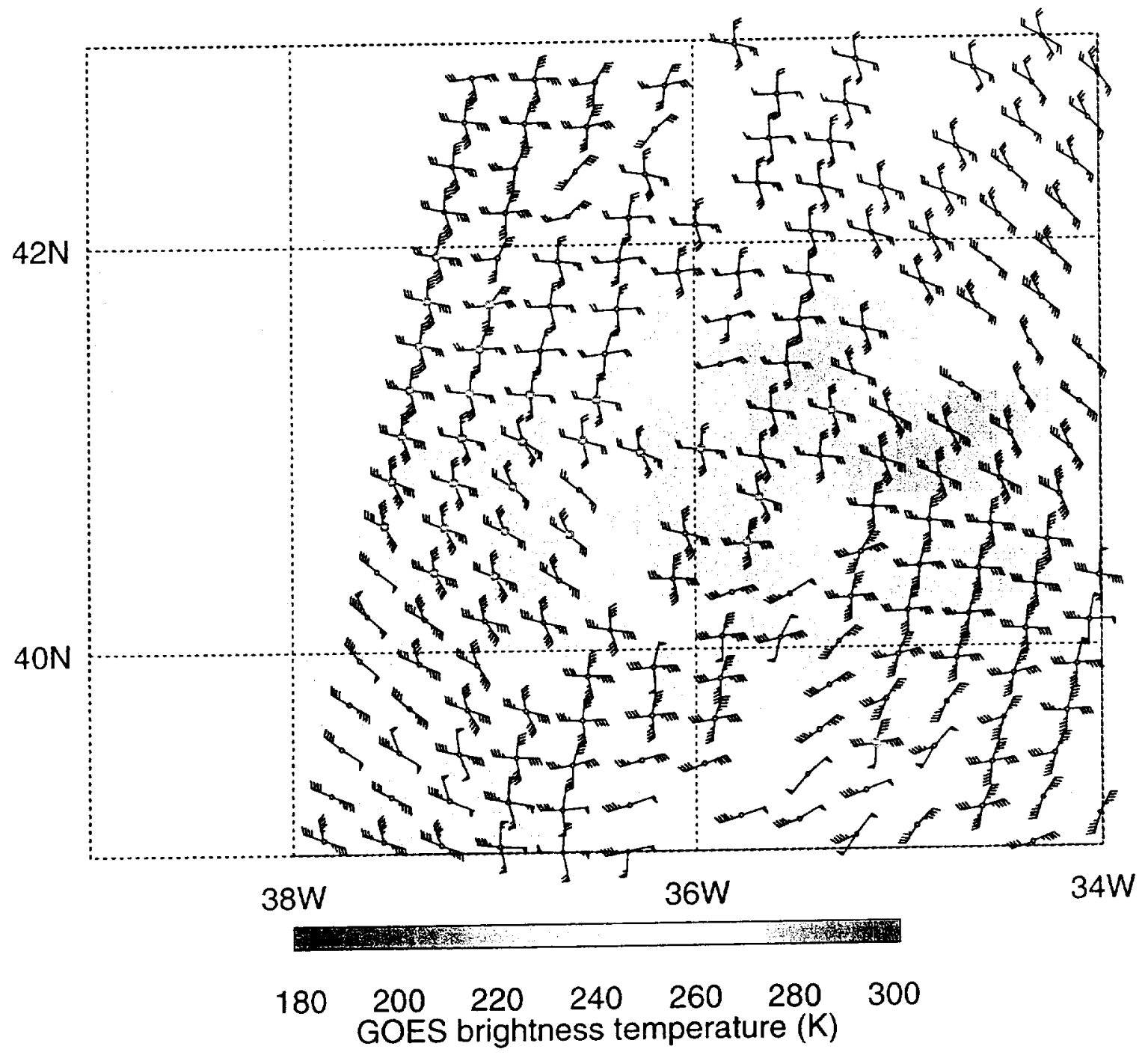


Fig. 22:

(a)
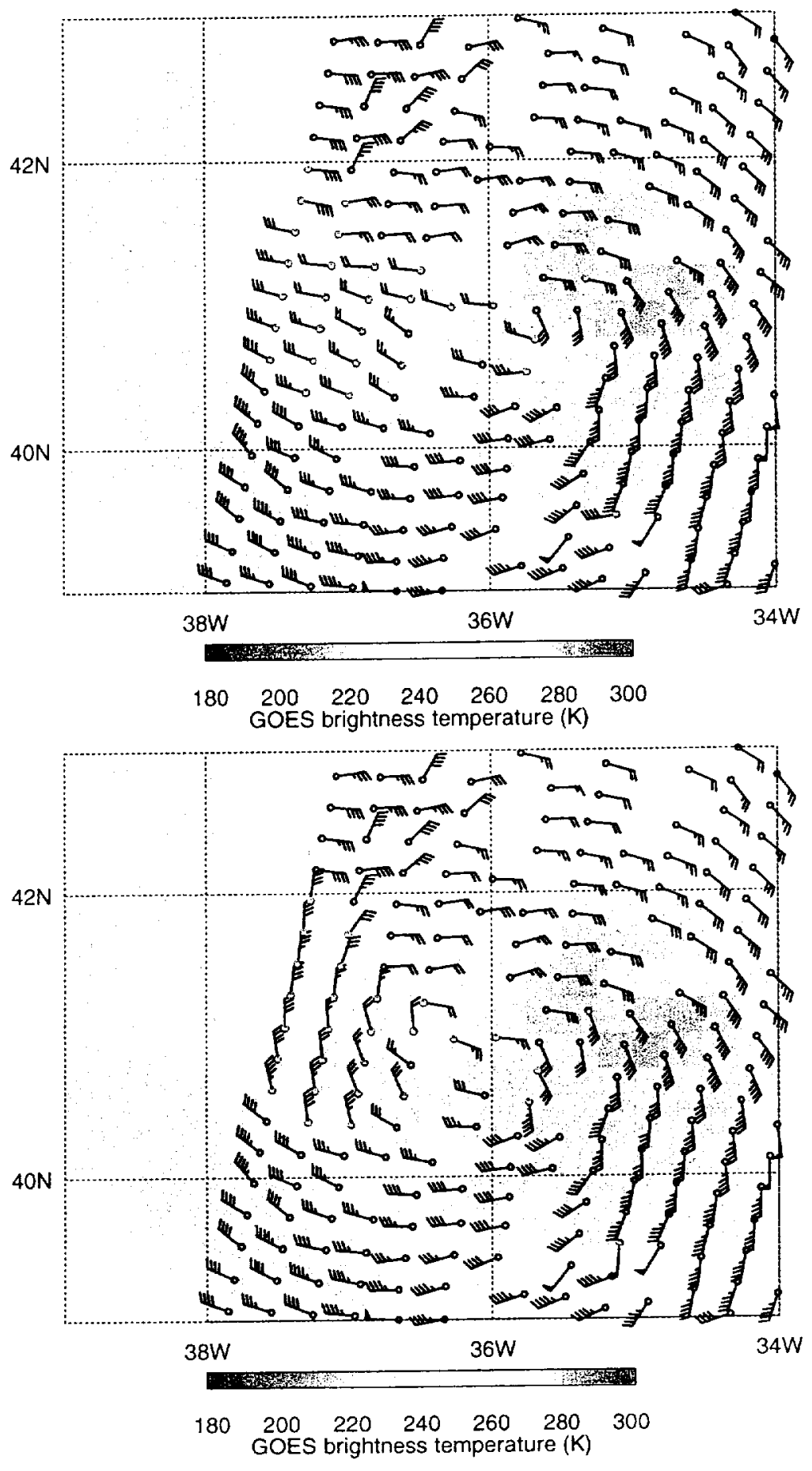